\title{
Ordering Cellular Spaces with Application to Curves and Knots
}

\author{
Anatoly B. Korchagin
}

Received: 27 November 2006 / Revised: 22 June 2007 /

Published online: 9 September 2007

(C) Springer Science+Business Media, LLC 2007

\begin{abstract}
This paper concerns the problem of ordering geometrical objects, which have the structure of finite cellular spaces. We introduce cellular codes, cellular indices, and $(k, l)$-sizes and apply them to ordering 2-cells, plane curves, and projections of knots. Results of this paper can be applied (1) to ordering other geometrical objects with cellular space structures, (2) in psychological and computer tests for comparison of complexity of geometrical objects, and (3) to ordering objects with fractal and chaotic structure, which admit approximation by cellular spaces.
\end{abstract}

Keywords Geometric complexity · Cellular spaces · Order relation · Curves · Knots and links $\cdot$ Real algebraic geometry

\section{Introduction}

A classification of geometric objects having topological or algebraic properties is usually accompanied by the problem of ordering classified objects. If the classification has enumerative nature, then this nature implies the order of objects. An example of such classification is the classification of smooth connected compact surfaces, where oriented surfaces can be ordered by numbers of holes and handles, and nonoriented surfaces - by numbers of holes and Möbius films.

If a classification does not have enumerative nature, then one can use an imagination of complexity of classified objects. An example of such order is the order of knots with $n$ crossings. There is generally accepted knot catalogue (see $[6,7]$ ) that has the standard notations, for example, the $7_{3}$ knot is the third knot with 7 crossings. This catalogue contains knots with 0 through 10 crossings. In our paper we order projections of knots with 5 through 9 crossings.

\footnotetext{
A.B. Korchagin $(\bowtie)$

Dept. of Math. and Statistics, Texas Tech. Univ., Lubbock, TX 79409-1042, USA

e-mail: anatoliy.korchagin@ttu.edu
} 
There exists an opinion that (see [1], p. 84) "There is no logic to the order in which we list the knots with a given crossing number. The first published catalogue of knots listed them in a certain order, and all subsequent catalogues have used the same arbitrary order." However in [1], the $7_{6}$ and $7_{7}$ knots together with their notations are interchanged with respect to generally accepted order. Even if there is no logic to the order in the knot catalogue, there is the impression that projections of knots were ordered like in a psychological test: from simple to more complicated picture.

In Sect. 6.2, we apply $(2,1)$-sizes (defined in Sect. 4), according to which projections of knots must be ordered in a specific way, and present their order for crossings from 5 through 9 in Table 2. Table 2 shows that the order in the knot catalogue and the order by $(2,1)$-sizes coincide for knots with 5 and 6 crossings. In Table 3 we calculate deviation of the order in the knot catalogue from the order by $(2,1)$-sizes for knots with 7, 8, and 9 crossings.

The initial point for writing this paper was the question how to order eight pictures shown in Fig. 6 of paper [2]. The same eight pictures are depicted in Fig. 15 of present paper. In paper [2], we proved the classification of $M$-curves of degree 5, where the arrangement of the odd branch of each $M$-curve with respect to two lines coincides with one of eight pictures depicted in Fig. 15. In paper [2], these pictures were arbitrary enumerated. In Fig. 15, they are ordered regarding to their $(2,1)$-sizes. If $1,2, \ldots, 8$ is the order of pictures in Fig. 15, then $1,4,3,2,7,8,5,6$ is the order of the same pictures in Fig. 6 of paper [2].

In Sect. 2 we define cellular codes and phenomenality of cellular spaces regarding to cellular codes. Phenomenality is an order relation, which reflects geometric complexity of cellular spaces. In Sect. 3 we give the classification of 2-cells with cellular codes $(1,0,1)$ and $(k, k, 1)$ for $k=1,2,3,4,5$. In Sect. 4 we define phenomenality of cellular spaces regarding to cellular indices and $(k, l)$-sizes. In Sect. 5 we define metrics in the set of permutations of integers $1,2, \ldots, k$. In Sect. 6, first, we apply $(2,1)$-sizes to order curves and knots, and second, we apply metrics from Sect. 5 to calculate deviation of the order in the knot catalogue from the order by $(2,1)$-sizes.

\section{Cellular Codes}

In this paper a cellular space is a Hausdorff $\mathrm{CW}$-complex. We consider only finite finite-dimensional cellular spaces, thus the words "cellular space" mean a finite $n$ dimensional cellular space with $0 \leq n<\infty$. We denote the closure of $r$-cell $e^{r}$ as $\mathrm{Cl} e^{r}$, and the $r$-skeleton of cellular space $X$ as sk $e_{r} X$.

Let $X$ be a cellular space, which partition consists of open $r$-cells $e_{1}^{r}, e_{2}^{r}, \ldots, e_{q_{r}}^{r}$ for $r=0,1,2, \ldots, n$. We choose one point $p_{i}^{r}$ in every cell $e_{i}^{r}$. Let $U_{i}^{r}$ be neighborhoods in $X$ of points $p_{i}^{r}$ satisfying the following conditions.

(i) For distinct points $p_{i}^{r}$ and $p_{j}^{s}$, their neighborhoods $U_{i}^{r}$ and $U_{j}^{s}$ are disjoint.

(ii) If $e \in \mathrm{sk} e_{n-1} X$ is a cell, and $e_{i}^{r} \cap \mathrm{Cl} e=\emptyset$, then $U_{i}^{r} \cap \mathrm{Cl} e=\emptyset$.

(iii) If $e \in \operatorname{sk} e_{n-1} X$ is a cell, and $e_{i}^{r} \cap \mathrm{Cl} e \neq \emptyset$, then intersection $U_{i}^{r} \cap \mathrm{Cl} e$ consists of one connected component.

(iv) Every neighborhood $U_{i}^{r}$ is contractible along itself in $X$. 
Definition 2.1 Neighborhoods $U_{i}^{r}$ of points $p_{i}^{r}$ satisfying conditions (i)-(iv) are called regular neighborhoods.

Definition 2.2 If cells of cellular space $X$ are equipped with their regular neighborhoods, then

(1) the number of connected components of the set $U_{i}^{r} \backslash$ sk $e_{n-1} X$ is called the multiplicity of $r$-cell $e_{i}^{r}$ and denoted as $\mu_{i}^{r}$,

(2) the number of connected components of the set $\bigcup_{i=1}^{q_{r}}\left(U_{i}^{r} \backslash\right.$ sk $\left.e_{n-1} X\right)$ is called the $r$-dimensional number of space $X$ and denoted by $m_{r}$,

(3) the $(n+1)$-tuple $\left(m_{0}, m_{1}, \ldots, m_{n}\right)$ is called the cellular code of space $X$,

(4) the number $m=m_{0}+m_{1}+\cdots+m_{n}$ is called the weight of space $X$.

Remarks 2.3 Definition 2.2 implies the following primary statements.

(1) Since multiplicity of every $n$-cell in $n$-dimensional space equals 1 , then $m_{n}=q_{n}$.

(2) $m_{r}=\sum_{i=1}^{q_{r}} \mu_{i}^{r}$.

(3) If an open $r$-cell with $r<n$ does not belong to the closure of the set $\bigcup_{i=1}^{q_{n}} e_{i}^{n}$, then the multiplicity of this $r$-cell equals 0 .

Examples 2.4 (1) If solid torus $X=S^{1} \times D^{2}$ is equipped with the partition, which consists of

one 0 -cell $e_{1}^{0}=$ ort $_{1} \times$ ort $_{1}$, where ort $1=(1,0)$, two 1 -cells $e_{1}^{1}=\operatorname{ort}_{1} \times\left(S^{1} \backslash \operatorname{ort}_{1}\right)$ and $e_{2}^{1}=\left(S^{1} \backslash\right.$ ort $\left._{1}\right) \times$ ort $_{1}$, two 2-cells $e_{1}^{2}=\operatorname{ort}_{1} \times \operatorname{Int} D^{2}$ and $e_{2}^{2}=\left(S^{1} \backslash\right.$ ort $\left._{1}\right) \times\left(S^{1} \backslash\right.$ ort $\left._{1}\right)$, and one 3-cell $e_{1}^{3}=\left(S^{1} \backslash \operatorname{ort}_{1}\right) \times \operatorname{Int} D^{2}$,

then $\mu_{1}^{0}=\mu_{1}^{1}=\mu_{1}^{2}=2, \mu_{2}^{1}=\mu_{2}^{2}=\mu_{1}^{3}=1$, and cellular space $X$ has code $(2,3,3,1)$.

(2) If the standard sphere $S^{n}$ is equipped with the standard partition, which consists of one 0 -cell ort $1=(1,0, \ldots, 0)$ and one $n$-cell $S^{n} \backslash$ ort $_{1}$, then cellular space $S^{0}$ has code (2); $S^{1}$ has code $(2,1)$; and for $n \geq 2, S^{n}$ has code $(1,0, \ldots, 0,1)$, where 0 is repeated $n-1$ times.

(3) If the standard disk $D^{n}$ is equipped with the standard partition, which consists of one 0 -cell ort 1 , one $(n-1)$-cell $e^{n-1}=S^{n-1} \backslash \operatorname{ort}_{1}$, and one $n$-cell Int $D^{n}$, then cellular space $D^{1}$ has code $(2,1) ; D^{2}$ has code $(1,1,1)$; and for $n \geq 3, D^{n}$ has code $(1,0, \ldots, 0,1,1)$, where 0 is repeated $n-2$ times.

(4) If projective space $\mathbb{R} P^{n}$ is equipped with the standard partition, which consists of $r$-cells $e^{r}=\mathbb{R} P^{r} \backslash \mathbb{R} P^{r-1}$, where $0 \leq r \leq n$, then cellular space $\mathbb{R} P^{n}$ has code $(2, \ldots, 2,1)$, where 2 is repeated $n$ times.

(5) If complex projective space $\mathbb{C} P^{n}$ is equipped with the standard partition, which consists of $2 r$-cells $e^{2 r}=\mathbb{C} P^{r} \backslash \mathbb{C} P^{r-1}$, where $0 \leq r \leq n$, then cellular space $\mathbb{C} P^{n}$ has code $(1,0, \ldots, 1,0,1)$, where fragment 1,0 is repeated $n$ times.

(6) If quaternion projective space $\mathbb{H} P^{n}$ is equipped with the standard partition, which consists of $4 r$-cells $e^{4 r}=\mathbb{H} P^{r} \backslash \mathbb{H} P^{r-1}$, where $0 \leq r \leq n$, then cellular space $\mathbb{H} P^{n}$ has code $(1,0,0,0, \ldots, 1,0,0,0,1)$, where fragment $1,0,0,0$ is repeated $n$ times.

(7) If the Cayley projective space $\mathbb{C a} P^{n}$ is equipped with the standard partition, which consists of $8 r$-cells $e^{8 r}=\mathbb{C a} P^{r} \backslash \mathbb{C a} P^{r-1}$, where $1 \leq r \leq n$, then cellular 
space $\mathbb{C a} P^{n}$ has the code $(1,0,0,0,0,0,0,0, \ldots, 1,0,0,0,0,0,0,0,1)$, where fragment $1,0,0,0,0,0,0,0$ is repeated $n$ times.

Consider nonempty $n$-dimensional cellular spaces $X, Y$, and $Z$ such that $X=$ $Y \cup Z$ and $Y \cap Z \subset$ sk $e_{n-1} X$.

Lemma 2.5 If an open $r$-cell $e_{i}^{r} \subset X$ with $r \leq n-1$ belongs both to sk $e_{n-1} Y$ and sk $e_{n-1} Z$, and the cell $e_{i}^{r}$ has multiplicities $\mu_{i}^{r}, v_{i}^{r}, \xi_{i}^{r}$ in $X, Y, Z$, respectively, then $\mu_{i}^{r}=v_{i}^{r}+\xi_{i}^{r}$.

Proof Let $U_{i}^{r}$ be a regular neighborhood of point $p_{i}^{r} \in e_{i}^{r}$ in space $X$. Then $U_{i}^{r} \cap Y$ and $U_{i}^{r} \cap Z$ are regular neighborhoods of point $p_{i}^{r}$ in spaces $Y$ and $Z$, respectively. The sets $A=\left(U_{i}^{r} \cap Y\right) \backslash$ sk $e_{n-1} Y$ and $B=\left(U_{i}^{r} \cap Z\right) \backslash$ sk $e_{n-1} Z$ are disjoint. Thus the number of connected components of the set $A \cup B=\left[\left(U_{i}^{r} \cap(Y \cup Z)\right] \backslash\right.$ sk $e_{n-1}(Y \cup Z)$ is equal to the sum of the numbers of connected components of the sets $A$ and $B$.

Corollary 2.6 (1) The sum of the $r$-dimensional numbers of spaces $Y$ and $Z$ is equal to the $r$-dimensional number of space $X$,

(2) the component-wise sum of cellular codes of spaces $Y$ and $Z$ is equal to the cellular code of space $X$,

(3) the sum of weights of spaces $Y$ and $Z$ is equal to the weight of space $X$.

Corollary 2.7 If $\left\{e_{1}^{n}, e_{2}^{n}, \ldots, e_{q_{n}}^{n}\right\}$ is the set of all $n$-cells of $n$-dimensional cellular space $X$, then

(1) the sum of $r$-dimensional numbers of closed cells $\mathrm{Cl} e_{1}^{n}, \mathrm{Cl} e_{2}^{n}, \ldots, \mathrm{Cl} e_{q_{n}}^{n}$ is equal to $r$-dimensional number of space $X$,

(2) the component-wise sum of cellular codes of $\mathrm{Cl} e_{1}^{n}, \mathrm{Cl} e_{2}^{n}, \ldots, \mathrm{Cl} e_{q_{n}}^{n}$ is equal to the cellular code of space $X$,

(3) the sum of weights of $\mathrm{Cl} e_{1}^{n}, \mathrm{Cl} e_{2}^{n}, \ldots, \mathrm{Cl} e_{q_{n}}^{n}$ is equal to the weight of space $X$.

Definition 2.8 Let $\left(m_{0}, m_{1}, \ldots, m_{n}\right)$ and $\left(m_{0}^{\prime}, m_{1}^{\prime}, \ldots, m_{n^{\prime}}^{\prime}\right)$ be $n$ - and $n^{\prime}$-tuples of nonnegative integers. If $n \leq n^{\prime}$, then we add to the $n$-tuple $n^{\prime}-n$ zeros and convert it into an $n^{\prime}$-tuple. The $n^{\prime}$-tuples $\left(m_{0}, m_{1}, \ldots, m_{n}, 0,0, \ldots, 0\right)$ and $\left(m_{0}^{\prime}, m_{1}^{\prime}, \ldots, m_{n^{\prime}}^{\prime}\right)$ are called edited.

The following two definitions establish an order relation in the set of cellular spaces.

Definition 2.9 Let $M=\left(m_{0}, m_{1}, \ldots, m_{n}\right)$ and $M^{\prime}=\left(m_{0}^{\prime}, m_{1}^{\prime}, \ldots, m_{n}^{\prime}\right)$ be edited $n$ tuples. If there exists an integer $i, 0 \leq i \leq n$, such that $m_{n}=m_{n}^{\prime}, m_{n-1}=m_{n-1}^{\prime}, \ldots$, $m_{i+1}=m_{i+1}^{\prime}$ and $m_{i}>m_{i}^{\prime}$, then $n$-tuple $M$ is said to be greater than $n$-tuple $M^{\prime}$, and we write $M>M^{\prime}$, otherwise the $n$-tuples are said to be equal, and we write $M=M^{\prime}$.

Definition 2.10 Let there be a rule that assigns to every cellular space $X$ a finite $n$-tuple $M$. If cellular spaces $X$ and $X^{\prime}$ have edited $n$-tuples $M$ and $M^{\prime}$, respectively, and $M>M^{\prime}$, then the space $X$ is said to be more phenomenal (regarding to their $n$-tuples) than the space $X^{\prime}$. If $M=M^{\prime}$, then $X$ and $X^{\prime}$ are called equiphenomenal (regarding to their $n$-tuples). 
Fig. 1 Classification of six face polyhedra
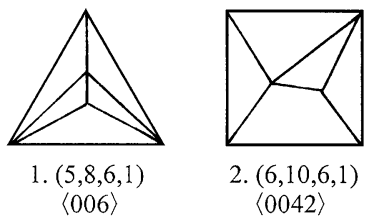

2. $(6,10,6,1)$ $\langle 0042\rangle$

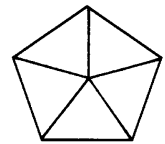

3. $(6,10,6,1)$ $\langle 00501\rangle$

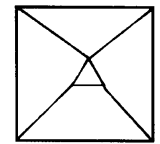

4. $(7,11,6,1)$ $\langle 0024\rangle$

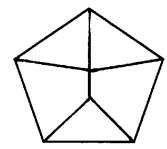

5. $(7,11,6,1)$ $\langle 00321\rangle$

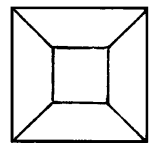

6. $(8,12,6,1)$ $\langle 0006\rangle$

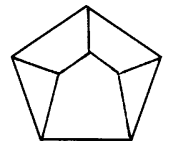

7. $(8,12,6,1)$ $\langle 00222\rangle$

Cellular codes can be subject to Definitions 2.8 and 2.9, and thus Definition 2.10 defines an order relation in the set of cellular spaces. Equiphenomenal cellular spaces (regarding to cellular code) are called equicodal. For $0 \leq n<k<\infty$, every $k$-dimensional cellular space is always more phenomenal (regarding to cellular code) than $n$-dimensional one.

Example 2.11 Every $(n+1)$-dimensional convex polyhedron has the natural cellular structure: its $i$-dimensional faces, $0 \leq i \leq n+1$, can be considered as $i$-cells. If $m_{0}, m_{1}, \ldots, m_{n}, 1$ are the numbers of its 0-cells (vertices), 1-cells (edges), .., $n$-cells (faces), and $(n+1)$-cell (one $(n+1)$-dimensional solid body), respectively, then it has cellular code $\left(m_{0}, m_{1}, \ldots, m_{n}, 1\right)$. Ordering Platonic solids regarding to their cellular codes coincides with well-known ordering: tetrahedron with the code $(4,6,4,1)$, cube $(8,12,6,1)$, octahedron $(6,12,8,1)$, dodecahedron $(20,30,12,1)$, and icosahedron $(12,30,20,1)$.

Example 2.12 This example is found in [5]. The net of a polyhedron is the union of its edges. Two polyhedra belong to the same type if the nets of their edges are homeomorphic. How many types of polyhedra with $q$ faces do there exist? The number $p$ of the types of polyhedra with $q$ faces is limited. If $q=4,5,6,7,8, \ldots$, then $p=1,2,7,34,257, \ldots$, respectively. In [5], one can find the classification for $q=4,5,6$ in pictures. In Fig. 1, one can see the classification of polyhedra for $q=6$, where each polyhedron is shown from above, and the frame for each picture is the base of the polyhedron. We order these polyhedra regarding to their cellular codes. In [5] they have the order $1,6,4,2,3,5,7$ and are ordered regarding to faces with maximal number of edges and pictured such that the face with maximal number of edges is placed in the base of the polyhedron. Notation $\langle\ldots\rangle$ in Fig. 1 is defined in Sect. 4.

\section{Equicodal Cellular Spaces}

Consider $n$-dimensional cellular space $X$. Let $Y$ and $Z$ be nonempty cellular equivalent $r$-dimensional $(r<n)$ cellular subspaces of $X$. Let $f: Y \rightarrow Z$ be a cellular homeomorphism, which realizes the cellular equivalence of $Y$ and $Z$, such that the restriction of $f$ on $W=Y \cap Z$ is the identity map, i.e. $\left.f\right|_{W}=i d_{W}$. This includes 
the case when $W=\emptyset$, since the condition $\left.f\right|_{\emptyset}=i d_{\emptyset}$ is empty. Let $\mathcal{F}$ be the partition, whose elements are pairs $(y, f(y))$ if $y \in Y \backslash W$ and single points of set $[X \backslash(Y \cup Z)] \cup W$. Let $X / \mathcal{F}$ be the quotient cellular space of $X$ by partition $\mathcal{F}$.

Theorem 3.1 Cellular spaces $X$ and $X / \mathcal{F}$ are equicodal, and if spaces $Y$ and $Z$ are nonempty, then $X$ and $X / \mathcal{F}$ realize distinct cellular equivalence classes.

Proof If $\mathrm{pr}: X \rightarrow X / \mathcal{F}$ is the quotient projection, then its restriction on the set $[X \backslash(Y \cup Z)] \cup W$ is a homeomorphism, say $h$. If $U_{1}$ and $U_{2}$ are regular neighborhoods of points $p$ and $f(p)$, respectively, then $\operatorname{pr}\left(U_{1} \cup U_{2}\right)$ is a regular neighborhood of the point $\operatorname{pr}(p)=\operatorname{pr}(f(p))$, and the sets $\left(U_{1} \cup U_{2}\right) \backslash$ ske $e_{n-1} X$ and $h\left(U_{1} \cup U_{2}\right) \backslash \operatorname{pr}(Y \cup Z)$ have the same number of connected components. If spaces $Y$ and $Z$ are nonempty, then the number of cells in $X / \mathcal{F}$ less than in $X$. This means that $X / \mathcal{F}$ and $X$ are not cellular equivalent.

There appear two natural problems.

Problem 1 Let $\left(m_{0}, m_{1}, \ldots, m_{n-1}, 1\right)$ be an $(n+1)$-tuple, $n \geq 0$, of nonnegative integers. Does there exist a closed $n$-cell which realizes this code?

Problem 2 What are cellular equivalence classes of cells realizing the same cellular code?

At first we discuss Problem 1 . There is no cell realizing a code with $m_{0}=0$, since the closure of every nonempty cell contains at least one 0 -cell, which has at least one connected component in the set $U_{i}^{r} \backslash$ sk $e_{n-1} X$ described in Definition 2.2.

The solution for 0 - and 1-cells is trivial. A 1-tuple $\left(m_{0}\right)$ is the code of a 0-cell iff $m_{0}=1$. A 2-tuple $\left(m_{0}, 1\right)$ is the code of a 1-cell iff $m_{0}=2$. There are two classes of equicodal 1-cells. One of them contains $S^{1}$, the other one contains $D^{1}$, and both of them are equipped with the standard partitions.

For $n \geq 2$, it is useful to have a dictionary of $n$-tuples which are realizable by closed cells. One can choose the set $\{1,2, \ldots\}$ of positive integers as the alphabet in the dictionary. The set of codes of $n$-cells constitutes in the dictionary an article of letter " $n$ ", which consists of $(n+1)$-tuple codes $\left(m_{0}, m_{1}, \ldots, m_{n-1}, 1\right)$. One can subdivide the article of letter " $n$ " in subarticles " $n, m$ " with $m=1,2, \ldots$, each of which contains, if any, codes with the same weight $m$. Inside the subarticles, codes may be lexicographically listed: if $M=\left(m_{0}, m_{1}, \ldots, m_{n-1}, 1\right)$ and $M^{\prime}=$ $\left(m_{0}^{\prime}, m_{1}^{\prime}, \ldots, m_{n-1}^{\prime}, 1\right)$ are distinct codes of two cells such that $\left(m_{0}, m_{1}, \ldots, m_{k-1}\right)=$ $\left(m_{0}^{\prime}, m_{1}^{\prime}, \ldots, m_{k-1}^{\prime}\right)$ for some $k=0,1, \ldots, n-1$, and $m_{k}<m_{k}^{\prime}$, then code $M^{\prime}$ follows after code $M$. Finally, each code-word can have several "meanings," which are cellular equivalence classes of cells. We denote these classes as $X_{m, i}^{n}$, where subscript index $i=1,2, \ldots$ enumerates the classes in some order. In this sense the article of letter " 0 " contains one subarticle " 0,1 " with one code (1), which has one "meaning" $\mathrm{X}_{1}^{0}=\mathrm{X}_{1,1}^{0}$, - the class of 0 -cell $D^{0}$. The article of letter " 1 " contains one subarticle "1, 3" with one code $(2,1)$, which has two "meanings" $\mathrm{X}_{3,1}^{1}=\left[D^{1}\right]$ and $\mathrm{X}_{3,2}^{1}=\left[S^{1}\right]$, where both $D^{1}$ and $S^{1}$ are equipped with the standard partitions. 
Lemma 3.2 There exist 2 -cells with codes $(1,0,1)$ and $(k, k, 1)$, where $k \geq 1$.

Proof Code $(1,0,1)$ is realized by sphere $S^{2}$ equipped with standard partition; code $(k, k, 1)$, where $k \geq 1$, is realized by disk $D^{2}$ equipped with the partition, which consists of $k$ distinct 0 -cells $e_{1}^{0}, \ldots, e_{k}^{0} \in S^{1}=D^{2} \backslash$ Int $D^{2}, k$ distinct 1-cells, which are connected components of the set $S^{1} \backslash\left\{e_{1}^{0}, \ldots, e_{k}^{0}\right\}$, and one 2-cell Int $D^{2}$.

Definition 3.3 The cellular space $D^{2}$ equipped with the partition described in the previous proof, which for $k \geq 1$, consists of $k 0$-cells, $k 1$-cells, and one 2-cell, is called the $k$-gon. Sphere $S^{2}$ with the standard partition is called a 0 -gon.

Lemma 3.4 The triples, which are distinct from $(1,0,1)$ and $(k, k, 1)$, where $k \geq 1$, are not realizable by 2-cells.

Proof It is obvious that there are no 2 -cells with codes $(0, l, 1)$ with $l \geq 0$. For the remaining triples, assume the contrary that for $k \geq 1, l \geq 0$, and $k \neq l$, there exists a 2-cell $X$ with code $(k, l, 1)$. Let the cellular partition of $X$ consists of 0 -cells $e_{1}^{0}, \ldots, e_{q_{0}}^{0}, 1$-cells $e_{1}^{1}, \ldots, e_{q_{1}}^{1}$, and the 2-cell $e^{2}$. Let att $: S^{1} \rightarrow$ sk $e_{1} X$ be a map which attaches the standard disk $D^{2}$ to 1 -skeleton sk $e_{1} X$. In the homotopy class of map att', one can choose a map att such that

(1) for each 0 -cell $e_{i}^{0}$ the set att ${ }^{-1}\left(e_{i}^{0}\right)$ consists of $\mu_{i}^{0}$ distinct points,

(2) for each 1-cell $e_{j}^{1}$ the set att ${ }^{-1}\left(e_{j}^{1}\right)$ consists of $\mu_{j}^{1}$ distinct connected components, say $D_{1}, \ldots, D_{\mu_{j}^{1}}$, homeomorphic to open segment $\operatorname{Int} D^{1}$, and

(3) for $s=1, \ldots, \mu_{j}^{1}$, restrictions att $\left.\right|_{D_{s}}: D_{s} \rightarrow \operatorname{att}\left(D_{s}\right)$ are homeomorphisms.

This means that the set att ${ }^{-1}\left(\left\{e_{1}^{0}, \ldots, e_{q_{0}}^{0}\right\}\right)$ consists of $k$ distinct points, and the set $\operatorname{att}^{-1}\left(\bigcup_{i=1}^{q_{1}} e_{i}^{1}\right)$ consists of $l$ connected components (homeomorphic to Int $\left.D^{1}\right)$. It is clear that the sets att ${ }^{-1}\left(\left\{e_{1}^{0}, \ldots, e_{q_{0}}^{0}\right\}\right)$ and att ${ }^{-1}\left(\bigcup_{i=1}^{q_{1}} e_{i}^{1}\right)$ are disjoint, constitute $S^{1}=\left(\operatorname{att}^{-1}\left(\left\{e_{1}^{0}, \ldots, e_{q_{0}}^{0}\right\}\right)\right) \cup\left(\operatorname{att}^{-1}\left(\bigcup_{i=1}^{q_{1}} e_{i}^{1}\right)\right)$, and provide a cellular partition of the circle $S^{1}$. Using this partition, one can find that the Euler characteristic of the circle $S^{1}$ is $k-l$. On the other hand the Euler characteristic of the circle is zero, and thus $k=l$. The contradiction proves the lemma.

Corollary 3.5 The article of letter " 2 " consists of subarticles " $2, m$ " with weights $m=2,3,5,7, \ldots$, each of which consists of one code $(1,0,1),(1,1,1),(2,2,1)$, $(3,3,1), \ldots$, respectively.

Return to Problem 2. We show that it is algorithmically solvable for $n=2$. The algorithm for the solution is the content of the proof of Theorem 3.6. But before dealing with this algorithm, we consider the first trivial cases and make some general remarks.

There is only one cellular equivalence class $\mathrm{X}_{2}^{2} \equiv \mathrm{X}_{2,1}^{2}$ of 2-cells with the code $(1,0,1)$. The representative of this class is the standard sphere $S^{2}$ equipped with the standard partition, which is a 0-gon. 
For each code $(k, k, 1)$ with $k \geq 1$, the cellular equivalence class which contains a $k$-gon is called the fundamental class of the code $(k, k, 1)$ and denoted as $\mathrm{X}_{2 k+1,1}^{2}$. The set of cellular equivalence classes of 2-cells with code $(1,1,1)$ consists of the unique class $\mathrm{X}_{3}^{2} \equiv \mathrm{X}_{3,1}^{2}$, which is fundamental.

If $k \geq 2$, then the set of cellular equivalence classes of 2-cells with code $(k, k, 1)$ consists of more than one class. One can construct their representatives by induction starting from a $k$-gon and by application to the $k$-gon the following two rules and their compositions. These rules are two special cases of Theorem 3.1: the first case is when $Y$ and $Z$ are two distinct 0-cells in $X$, and the second case is when $Y$ and $Z$ are two distinct cellular equivalent 1-cells in $X$. Let $X$ be a 2-cell with code $(k, k, 1)$, which realizes cellular equivalence class $\mathbf{X}_{2 k+1, i}^{2}$.

Rule \#1 If the cellular partition of $X$ contains at least two 0-cells, say $e_{1}$ and $e_{2}$, then quotient cellular space $X /\left(e_{1} \cup e_{2}\right)$ is a representative of a class $X_{2 k+1, j}^{2}$ of the same code $(k, k, 1)$, which is different from the class $\mathrm{X}_{2 k+1, i}^{2}$.

Rule \#2 If the cellular partition of $X$ contains at least two cellular equivalent 1-cells, say $e_{1}$ and $e_{2}$, and $f:\left(\mathrm{Cl} e_{1}, \operatorname{sk} e_{0} \mathrm{Cl} e_{1}\right) \rightarrow\left(\mathrm{Cl} e_{2}, \operatorname{sk} e_{0} \mathrm{Cl} e_{2}\right)$ is the cellular homeomorphism, then quotient cellular space $X /\left(\bigsqcup_{x \in \mathrm{Cl} e_{1}}(x \cup f(x))\right.$ is a representative of a class $\mathrm{X}_{2 k+1, j}^{2}$ of the same code $(k, k, 1)$, which is different from the class $\mathrm{X}_{2 k+1, i}^{2}$.

Remark about figures Using the previous Rules, we generate from a $k$-gon representatives of cellular equivalence classes of 2-cells with code $(k, k, 1)$. If, under application of Rule \#1 or \#2, a vertex is not identified with another one, then we do not mark the vertex and draw it like <. If, under application of Rule \#1 or \#2, two or more vertices are identified, then we mark all of them either by the bullet $\bullet$ or by the circle $\propto<$. If, under application of Rule \#2, an edge is not identified with another one, then we draw it as a usual segment —. If, under application of Rule \#2, two or more edges are identified, then we mark all of them either by the arrow $\longrightarrow$ or by the double arrow $\longrightarrow$ (see Figs. 2-6). Moreover, the arrows define orientations of the edges, according to which the identification is made.

We use the following symbols for identifications of vertices and edges. Symbol 0 means that we do not identify vertices and edges. Let the numbers of 0-cells and 1 -cells of a 2-cell be equal to $p$ and $q$, respectively.

If for $\alpha \geq 1$, integers $p_{1}, \ldots, p_{\alpha}, p$ satisfy inequalities $2 \leq p_{1} \leq \cdots \leq p_{\alpha}$ and $p_{1}+\cdots+p_{\alpha} \leq p$, then symbol $V\left(p_{1}, p_{2}, \ldots, p_{\alpha}\right)$ means that first, we identify $p_{1}$ vertices, then we identify another $p_{2}$ vertices, ..., and finally we identify another $p_{\alpha}$ vertices.

Fig. 2 Cellular equivalence classes of the code $(2,2,1)$

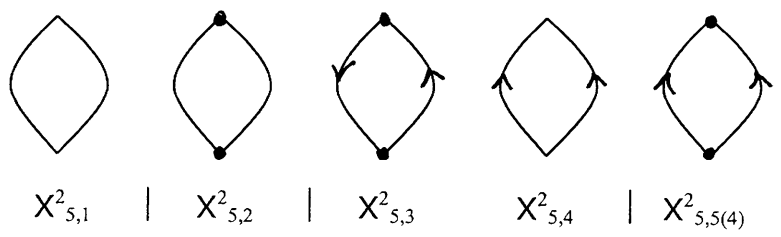


Fig. 3 Cellular equivalence classes of the code $(3,3,1)$
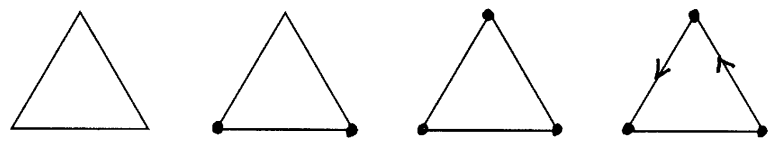

$$
\mathrm{X}^{2}{ }_{7,1}
$$$$
\mathrm{X}^{2}{ }_{7,2}
$$

$X^{2} 7,3$

$\mathrm{X}^{2}{ }_{7,4}$
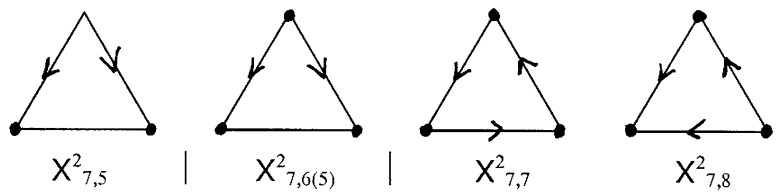
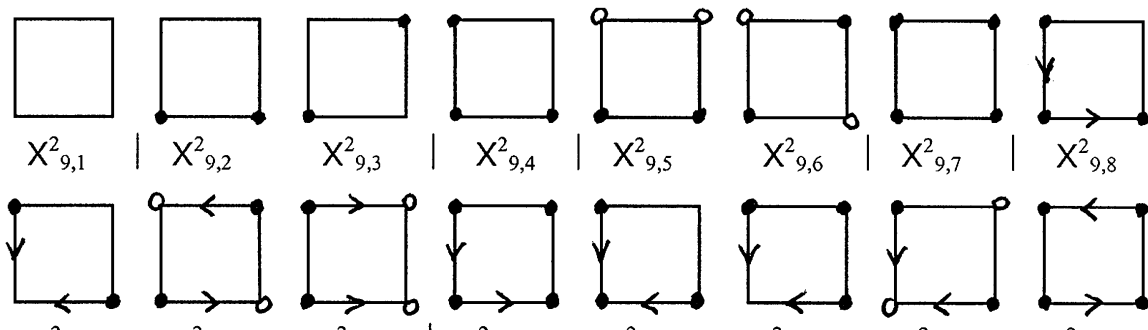

$\mathrm{X}^{2} 9,13(9)$

$\mathrm{X}^{2} 9,14(9)$

$\mathrm{X}^{2} 9,15(9)$
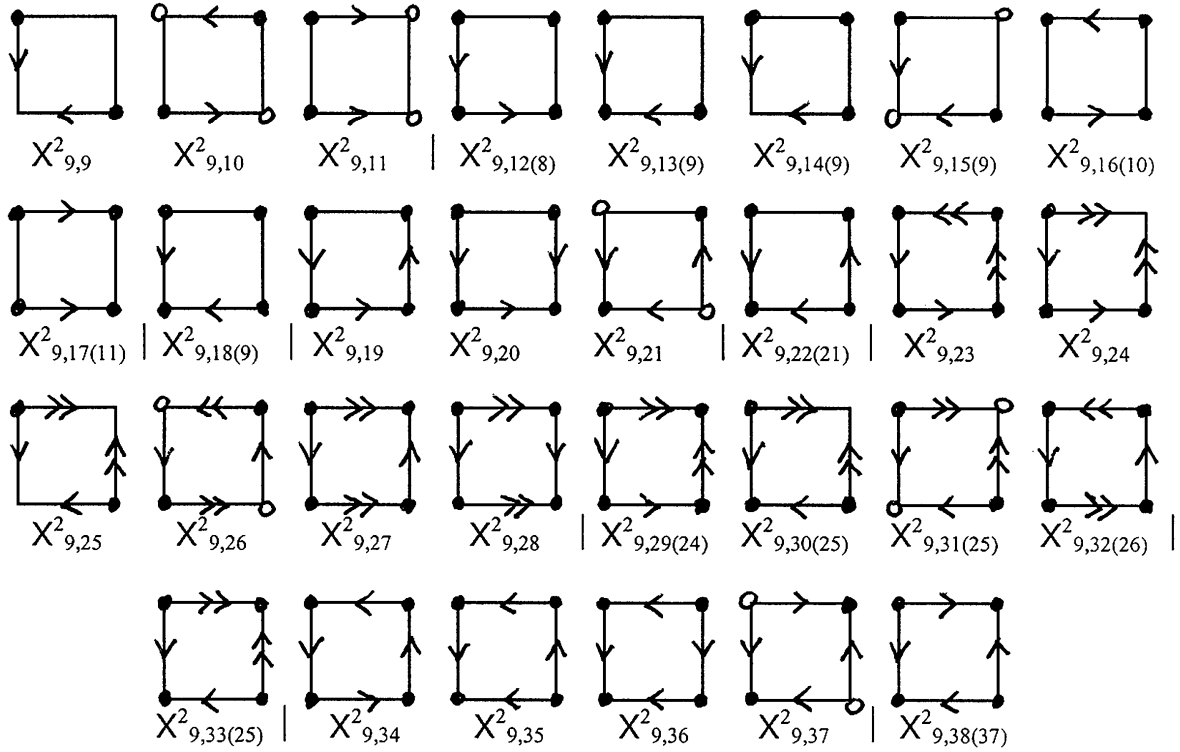

Fig. 4 Cellular equivalence classes of the code $(4,4,1)$

If for $\beta \geq 1$ integers $q_{1}, \ldots, q_{\beta}, q$ satisfy inequalities $2 \leq q_{1} \leq \cdots \leq q_{\beta}$ and $q_{1}+$ $\cdots+q_{\beta} \leq q$, then symbol $E\left(q_{1}, q_{2}, \ldots, q_{\beta}\right)$ means that first, we identify $q_{1}$ edges, then we identify another $q_{2}$ edges, ..., and finally we identify another $q_{\beta}$ edges.

If we have identified edges of the fundamental $k$-gon by means of symbol $E\left(q_{1}, \ldots, q_{\beta}\right)$ and obtained a closed 2-cell $\mathrm{Cl} e$ having a number $p \geq 2$ of 0 -cells, then for any integers $2 \leq p_{1} \leq \cdots \leq p_{\alpha}$ and $p_{1}+\cdots+p_{\alpha} \leq p$, symbol $\left(E\left(q_{1}, \ldots, q_{\beta}\right), V\left(p_{1}, \ldots, p_{\alpha}\right)\right)$ means that we identify vertices of 2 -cell $\mathrm{Cl} e$ by means of symbol $V\left(p_{1}, \ldots, p_{\alpha}\right)$. 

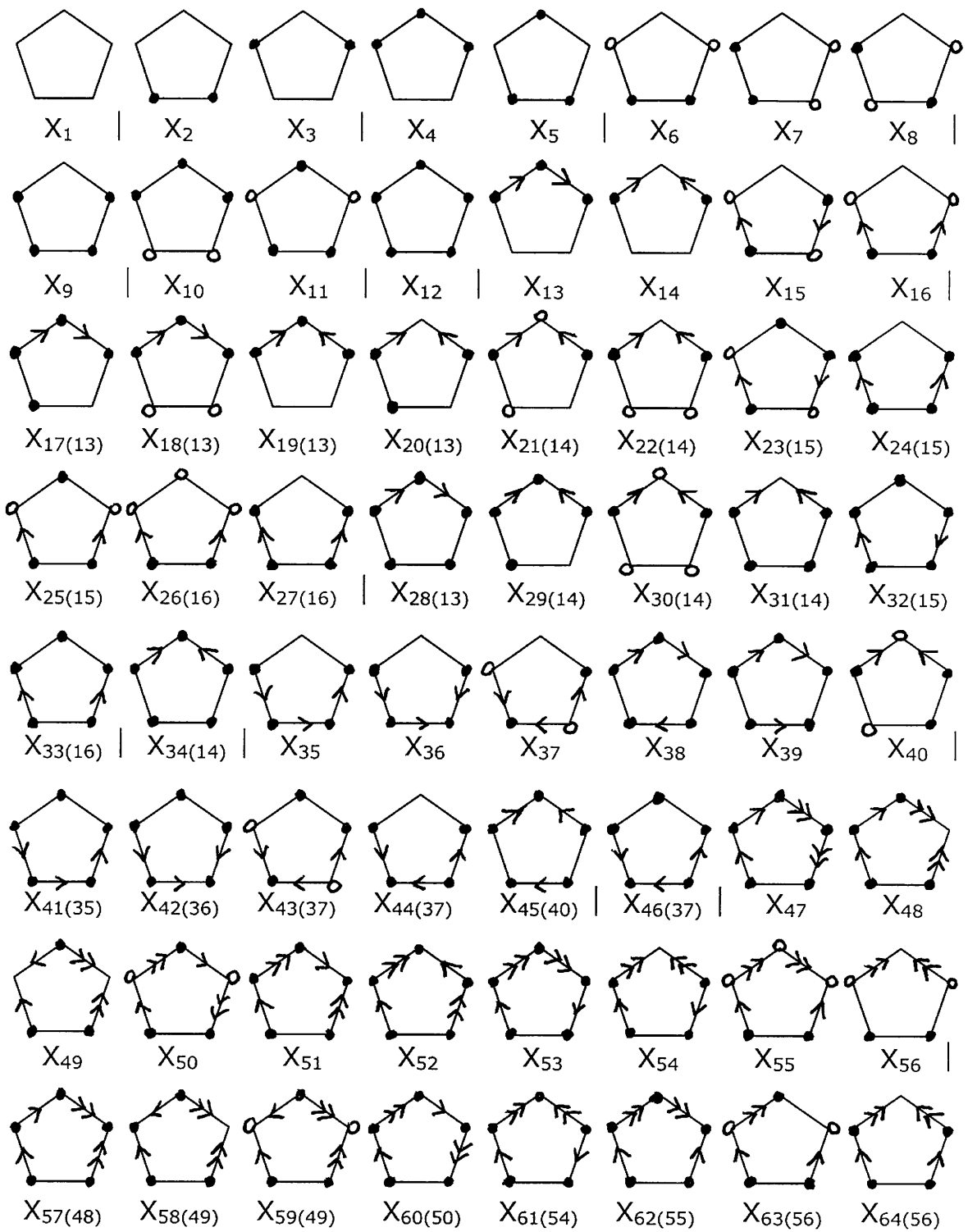

Fig. 5 Cellular equivalence classes of the code $(5,5,1)$ (beginning)

We apply the following two abbreviations: $\left(0, V\left(p_{1}, \ldots, p_{\alpha}\right)\right)=V\left(p_{1}, \ldots, p_{\alpha}\right)$ and $\left(E\left(q_{1}, \ldots, q_{\beta}\right), 0\right)=E\left(q_{1}, \ldots, q_{\beta}\right)$. In Figs. $1-5$, vertical bar $\mid$ is used to separate identifications with distinct symbol.

Since every identification of two or more edges is automatically accompanied by some identification of their terminal points, symbol $\left(V\left(p_{1}, \ldots, p_{\alpha}\right), E\left(q_{1}, \ldots, q_{\beta}\right)\right)$, where both of $V\left(p_{1}, \ldots, p_{\alpha}\right)$ and $E\left(q_{1}, \ldots, q_{\beta}\right)$ are nonzero, is prohibited. 


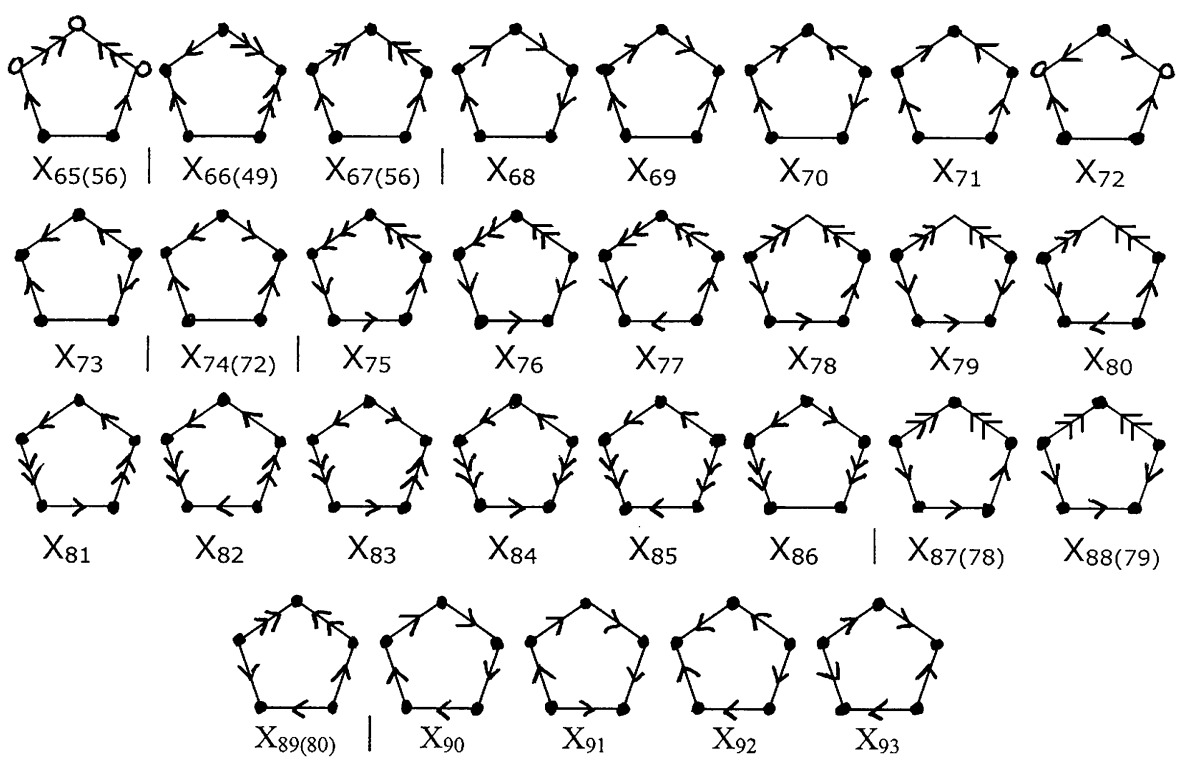

Fig. 6 Cellular equivalence classes of the code $(5,5,1)$ (the end)

In Figs. 2-6, we use the following notations. Notation $i(j)$, where $i>j$, in the index of class $\mathrm{X}_{2 k+1, i(j)}^{2}$ means that the representative of this class is obtained from class $\mathrm{X}_{2 k+1, j}^{2}$ by means of identification of vertices or edges indicated in the picture of this class. If $j=1$, then we reduce notation $\mathrm{X}_{2 k+1, i(1)}^{2}$ to $\mathrm{X}_{2 k+1, i}^{2}$. In Figs. 4 and 5 , we use abbreviation $\mathrm{X}_{11, i}^{2}=\mathrm{X}_{i}$ and $\mathrm{X}_{11, i(j)}^{2}=\mathrm{X}_{i(j)}$.

Theorem 3.6 There do not exist cellular equivalence classes of cellular spaces

(1) with the code $(1,0,1)$ other than the class, whose representative is the sphere $S^{1}$ equipped with the standard partition,

(2) with the code $(1,1,1)$ other than the class, whose representative is the disk $D^{1}$ equipped with the standard partition,

(3) with the code $(2,2,1)$ other than the five classes depicted in Fig. 2,

(4) with the code $(3,3,1)$ other than the eight classes depicted in Fig. 3,

(5) with the code $(4,4,1)$ other than the 38 classes depicted in Fig. 4,

(6) with the code $(5,5,1)$ other than the 93 classes depicted in Figs. 5 and 6.

Proof The statements of the items (1) and (2) are trivial. The proof of items (3) and (4) is simple; and one can just follow Figs. 2 and 3.

To order classes in Figs. 4-6, we use the following agreements.

(a) The fundamental class $\mathrm{X}_{2 k+1,1}^{2}$ has symbol 0 and is the first in ordering classes. (For the rest of the rule we consider only nonzero symbols.)

(b) Every class with symbol $V\left(p_{1}, \ldots, p_{\alpha}\right)$ is followed by the class with the symbol $E\left(q_{1}, \ldots, q_{\beta}\right)$. 
Table 1

\begin{tabular}{|c|c|c|c|c|c|c|c|}
\hline \# & $\begin{array}{l}\text { Symbol of } \\
\text { identifica- } \\
\text { tion }\end{array}$ & Classes $\mathrm{X}_{9, i}^{2}$ & Classes $\mathrm{X}_{11, i}^{2}$ & \# & $\begin{array}{l}\text { Symbol of } \\
\text { identifica- } \\
\text { tion }\end{array}$ & Classes $\mathrm{X}_{9, i}^{2}$ & Classes $\mathrm{X}_{11, i}^{2}$ \\
\hline 1 & 0 & $x_{9,1}^{2}$ & $x_{11,1}^{2}$ & 12 & $E(3)$ & $X_{9,19}^{2}-X_{9,21}^{2}$ & $x_{11,35}^{2}-x_{11,40}^{2}$ \\
\hline 2 & $V(2)$ & $x_{9,2}^{2}, x_{9,3}^{2}$ & $x_{11,2}^{2}, x_{11,3}^{2}$ & 13 & $(E(3), V(2))$ & $x_{9,22}^{2}$ & $X_{11,41}^{2}-X_{11,45}^{2}$ \\
\hline 3 & $V(3)$ & $x_{9,4}^{2}$ & $X_{11,4}^{2}, X_{11,5}^{2}$ & 14 & $(E(3), V(3))$ & & $x_{11,46}^{2}$ \\
\hline 4 & $V(2,2)$ & $x_{9,5}^{2}, x_{9,6}^{2}$ & $x_{11,6}^{2}-x_{11,8}^{2}$ & 15 & $E(2,2)$ & $X_{9,23}^{2}-X_{9,28}^{2}$ & $x_{11,47}^{2}-x_{11,56}^{2}$ \\
\hline 5 & $V(4)$ & $x_{9,7}^{2}$ & $x_{11,9}^{2}$ & 16 & $(E(2,2), V(2))$ & $x_{9,29}^{2}-x_{9,32}^{2}$ & $x_{11,57}^{2}-x_{11,65}^{2}$ \\
\hline 6 & $V(2,3)$ & & $x_{11,10}^{2}, x_{11,11}^{2}$ & 17 & $(E(2,2), V(3))$ & $x_{9,33}^{2}$ & $x_{11,66}^{2}-x_{11,67}^{2}$ \\
\hline 7 & $V(5)$ & & $x_{11,12}^{2}$ & 18 & $E(4)$ & $x_{9,34}^{2}-x_{9,37}^{2}$ & $X_{11,68}^{2}-X_{11,73}^{2}$ \\
\hline 8 & $E(2)$ & $x_{9,8}^{2}-x_{9,11}^{2}$ & $X_{11,13}^{2}-X_{11,16}^{2}$ & 19 & $(E(4), V(2))$ & $x_{9,38}^{2}$ & $\mathrm{X}_{11,74}^{2}$ \\
\hline 9 & $(E(2), V(2))$ & $X_{9,12}^{2}-X_{9,17}^{2}$ & $X_{11,17}^{2}-X_{11,27}^{2}$ & 20 & $E(2,3)$ & & $x_{11,75}^{2}-x_{11,86}^{2}$ \\
\hline 10 & $(E(2), V(3))$ & $x_{9,18}^{2}$ & $X_{11,28}^{2}-X_{11,33}^{2}$ & 21 & $(E(2,3), V(3))$ & & $X_{11,87}^{2}-X_{11,89}^{2}$ \\
\hline 11 & $(E(2), V(4))$ & & $x_{11,34}^{2}$ & 22 & $V(5)$ & & $X_{11,90}^{2}-X_{11,93}^{2}$ \\
\hline
\end{tabular}

(c) If $p_{1}+\cdots+p_{\alpha}<p_{1}^{\prime}+\cdots+p_{\alpha^{\prime}}^{\prime}$ (resp., $\left.q_{1}+\cdots+q_{\beta}<q_{1}^{\prime}+\cdots+q_{\beta^{\prime}}^{\prime}\right)$, then the class with symbol $V\left(p_{1}, \ldots, p_{\alpha}\right)$ (resp., $\left.E\left(q_{1}, \ldots, q_{\beta}\right)\right)$ is followed by the class with symbol $V\left(p_{1}^{\prime}, \ldots, p_{\alpha^{\prime}}^{\prime}\right)$ (resp., $\left.E\left(q_{1}^{\prime}, \ldots, q_{\beta^{\prime}}^{\prime}\right)\right)$.

(d) If, first, $\alpha=\alpha^{\prime}$ (resp., $\beta=\beta^{\prime}$ ), second, for some $\gamma<\alpha$ we have $p_{i}=p_{i}^{\prime}$ with $i=$ $1,2, \ldots, \gamma$ (resp., for some $\delta<\beta$ we have $q_{i}=q_{i}^{\prime}$ with $i=1,2, \ldots, \delta$ ), and third, $p_{i+1}<p_{i+1}^{\prime}$ (resp., $\left.q_{i+1}<q_{i+1}^{\prime}\right)$, then the class with symbol $V\left(p_{1}, \ldots, p_{\alpha}\right)$ (resp., $\left.E\left(q_{1}, \ldots, q_{\beta}\right)\right)$ is followed by the class with symbol $V\left(p_{1}^{\prime}, \ldots, p_{\alpha^{\prime}}^{\prime}\right)$ (resp., $\left.E\left(q_{1}^{\prime}, \ldots, q_{\beta^{\prime}}^{\prime}\right)\right)$.

(e) If the classes with symbols $E\left(q_{1}, \ldots, q_{\beta}\right)$ and $E\left(q_{1}^{\prime}, \ldots, q_{\beta^{\prime}}^{\prime}\right)$ satisfy condition of item (c) or item (d), then the class with symbol $\left(E\left(q_{1}, \ldots, q_{\beta}\right), V\left(p_{1}, \ldots, p_{\alpha}\right)\right)$ is followed by the class with symbol $\left(E\left(q_{1}^{\prime}, \ldots, q_{\beta^{\prime}}^{\prime}\right), V\left(p_{1}^{\prime}, \ldots, p_{\alpha^{\prime}}^{\prime}\right)\right)$.

(f) If $E\left(q_{1}, \ldots, q_{\beta}\right)=E\left(q_{1}^{\prime}, \ldots, q_{\beta^{\prime}}^{\prime}\right)$ and the classes with symbols $V\left(p_{1}, \ldots, p_{\alpha}\right)$ and $V\left(p_{1}^{\prime}, \ldots, p_{\alpha^{\prime}}^{\prime}\right)$ satisfy condition of item (c) or item $(\mathrm{d})$, then the class with symbol $\left(E\left(q_{1}, \ldots, q_{\beta}\right), V\left(p_{1}, \ldots, p_{\alpha}\right)\right)$ is followed by the class with symbol $\left(E\left(q_{1}^{\prime}, \ldots, q_{\beta^{\prime}}^{\prime}\right), V\left(p_{1}^{\prime}, \ldots, p_{\alpha^{\prime}}^{\prime}\right)\right)$.

To complete the proof we present the Table 1, which helps to generate the cellular equivalence classes indicated in Figs. 4-6.

The scheme of the proof can be applied to ordering cellular spaces of codes $(k, k, 1)$ with $k \geq 6$.

Definition 3.7 A cellular space is said to be saturated if every two of its distinct subspaces are not cellular equivalent. The cellular equivalence class of a saturated space is also called saturated.

Classes $X_{3,2}^{1}, X_{2}^{2}, X_{3}^{2}, X_{5,3}^{2}, X_{5,5}^{2}, X_{7,7}^{2}-X_{7,8}^{2}, X_{9,34}^{2}-X_{9,36}^{2}, X_{9,38}^{2}$, and $X_{11,90}^{2}-X_{11,93}^{2}$ are saturated. It is clear that one can not apply Rules \#1 and \#2 to the representatives of saturated classes. 
Let $A_{1}, \ldots, A_{l}$ be the set of all symbols obtained by identification of vertices and edges of a $k$-gon, and let $h_{k}\left(A_{i}\right)$ be the number of cellular equivalent classes corresponding to symbol $A_{i}$. What is the number $h_{k}\left(A_{i}\right)$ of cellular equivalence classes corresponding to symbols $A_{i}$ ? What is the total number of cellular equivalence classes of a $k$-gon? It is trivial that $h_{k}(0)=1$ for all $k \geq 1$, and it is easily to prove that $h_{k}(V(2))=\left[\frac{k}{2}\right], h_{k}(V(3))=\sum_{i=1}^{\left[\frac{k+1}{3}\right]}\left[\frac{k-3 i-1}{2}\right]$, and $h_{k}(E(2))=2 h_{k}(V(2))$, where $[a]$ means the greatest integer in $a$.

Conjecture 3.8 The total number of cellular equivalence classes of a $k$-gon is odd if $k \equiv 1$ or $2 \bmod 4$ and is even if $k \equiv 0$ or $3 \bmod 4$.

Remarks 3.9 (1) If dimension $n \geq 1$, then there is no $n$-cell with weight 1 .

(2) If $n \geq 2$, then there is the unique code $(1,0, \ldots, 0,1)$ having weight $m=2$. This code represents the unique cellular equivalence class, whose representative is the standard sphere $S^{n}$ equipped with the standard partition.

(3) If $n \geq 2$, then there is the unique code $(1,0, \ldots, 0,1,1)$ having weight $m=3$. This code represents the unique cellular equivalence class, whose representative is the standard disk $D^{n}$ equipped with the standard partition.

Conjecture 3.10 There are no $n$-cells, $0 \leq n<\infty$, with even weight $m \geq 4$.

\section{Ordering Equiindexial Spaces}

Definition 4.1 An $n$-dimensional cellular space is said to have the cellular index $\left[i_{0}, i_{1}, \ldots, i_{n}\right]$ if the numbers of its $0-, 1-, \ldots, n$-cells are equal to $i_{0}, i_{1}, \ldots, i_{n}$, respectively.

If $M=\left[i_{0}, i_{1}, \ldots, i_{n}\right]$ and $M^{\prime}=\left[i_{0}^{\prime}, i_{1}^{\prime}, \ldots, i_{n^{\prime}}^{\prime}\right]$ are indices of spaces $X$ and $X^{\prime}$, respectively, and $n<n^{\prime}$, then we edit and order them according to Definitions 2.8 and 2.9. We assign to each cellular space its index.

If cellular spaces $X$ and $X^{\prime}$ have edited indices $M$ and $M^{\prime}$, respectively, and $M>M^{\prime}$, then according to Definition 2.10, the space $X$ is more phenomenal regarding to their indices than the space $X^{\prime}$, and we write $X \succ X^{\prime}$ or $X^{\prime} \prec X$. Equiphenomenal cellular spaces (regarding to their indices) are called equiindexial, and we write $X \asymp X^{\prime}$.

The phenomenality regarding to cellular index is an order relation on the set of cellular spaces. For $0 \leq m<n<\infty$ every $n$-dimensional space is always more phenomenal, than $m$-dimensional one.

Examples 4.2 (1) If an $(n+1)$-dimensional convex polyhedron, equipped with its natural cellular structure, has cellular code $\left(m_{0}, m_{1}, \ldots, m_{n}, 1\right)$, then its index is $\left[m_{0}, m_{1}, \ldots, m_{n}, 1\right]$ (in particular, a $k$-gon has index $[k, k, 1]$ ). Thus the cellular code phenomenality and index phenomenality define the same order relation on the set of convex polyhedra.

(2) All saturated 2-cells of all cellular codes $(k, k, 1)$ with $k \geq 1$ are equiindexial 2-cells with index $[1,1,1]$. 
(3) Sequences $X_{5,1}^{2} \succ X_{5,2}^{2} \succ X_{5,4}^{2} \succ X_{5,3}^{2} \asymp X_{5,5}^{2}$ and $X_{7,1}^{2} \succ X_{7,2}^{2} \succ X_{7,3}^{2} \succ X_{7,5}^{2} \succ$ $\mathrm{X}_{7,4}^{2} \asymp \mathrm{X}_{7,6}^{2} \succ \mathrm{X}_{7,7}^{2} \asymp \mathrm{X}_{7,8}^{2}$ represent the index phenomenality in the sets of cellular equivalence classes with cellular codes $(2,2,1)$ and $(3,3,1)$, respectively. In similar way, one order cellular equivalence classes with cellular codes $(4,4,1)$ and $(5,5,1)$.

If $n$-dimensional cellular space $X$ is comprised for $k=0,1,2, \ldots, n$ of $k$-cells $e_{1}^{k}, e_{2}^{k}, \ldots, e_{q_{k}}^{k}$, then the space $X$ has index $\left[q_{0}, q_{1}, \ldots, q_{n}\right]$. Let $(k+1)$-tuples, which are rows in the $\left[(k+1) \times q_{k}\right]$-matrix

$$
\left[\begin{array}{ccccc}
q_{01} & q_{11} & \cdots & q_{k-1,1} & 1 \\
q_{02} & q_{12} & \cdots & q_{k-1,2} & 1 \\
\cdots & \cdots & \cdots & \cdots & \cdots \\
q_{0 q_{k}} & q_{1 q_{k}} & \cdots & q_{k-1, q_{k}} & 1
\end{array}\right],
$$

be indices of closed $k$-cells of space $X$. The matrix is called the generalized $k$-dimensional cellular index (or $k$-index) of space $X$.

Let $s_{l}(p)$ be the number of terms, which are equal to $p$, in the $l$-th column of the matrix, $p=0,1, \ldots$, and $0 \leq l \leq k$. In particular, $s_{k}(1)=q_{k}$ and $s_{k}(p)=0$ if $p \geq 2$. It is clear that there is a finite number of nonzero terms in the sequence $s_{l}(0), s_{l}(1), \ldots, s_{l}(p), \ldots$ Let $p_{k l}$ be the integers, for which $s_{l}\left(p_{k l}\right) \neq 0$ and $s_{l}(p)=0$ if $p>p_{k l}$. It is obvious that $\sum_{p=1}^{p_{k l}} s_{l}(p)=\sum_{i=1}^{q_{k}} q_{l i}$.

Definition 4.3 Under the previous conditions, the $n$-dimensional cellular space $X$ is said to have (cellular) $(k, l)$-size $\left\langle s_{l}(0), s_{l}(1), \ldots, s_{l}\left(p_{k l}\right)\right\rangle_{k l}$.

If $\left\langle s_{l}(0), s_{l}(1), \ldots, s_{l}\left(p_{k l}\right)\right\rangle_{k l}$ and $\left\langle s^{\prime}(0), s^{\prime}(1), \ldots, s^{\prime}\left(p_{k l}^{\prime}\right)\right\rangle_{k l}$ are $(k, l)$-sizes of spaces $X$ and $X^{\prime}$, respectively, and $p_{k l}<p_{k l}^{\prime}$, then we subject them to Definitions 2.8 and 2.9 .

If cellular spaces $X$ and $X^{\prime}$ have edited $(k, l)$-sizes $M$ and $M^{\prime}$, respectively, and $M>M^{\prime}$, then according to Definition 2.10, the space $X$ is more phenomenal regarding to their $(k, l)$-sizes than the space $X^{\prime}$. If $M=M^{\prime}$, then $X$ and $X^{\prime}$ are said to have the same $(k, l)$-size phenomenality.

If either (1) space $X$ is more phenomenal than space $X^{\prime}$ regarding to their $(k, k-1)$-sizes, or (2) spaces $X$ and $X^{\prime}$ have the same $(k, l)$-size phenomenality for $l=k-1, k-2, \ldots, t+1$, and $X$ is more phenomenal than $X^{\prime}$ regarding to their $(k, t)$-sizes, then according to Definition 2.10, space $X$ is more phenomenal regarding to their $k$-indices than space $X^{\prime}$. If spaces $X$ and $X^{\prime}$ have the same $(k, l)$-size phenomenality for $l=k-1, k-2, \ldots, 1,0$, then spaces $X$ and $X^{\prime}$ are said to have the same $k$-index phenomenality.

Remarks 4.4 (1) Every 0-index of nonempty cellular space $X$ is a column matrix, whose elements are ones; thus every $(0,0)$-size is a 2-tuple $\left\langle 0, s_{0}(1)\right\rangle_{00}$, where $s_{0}(1)=q_{0}$ is the number of 0 -cells of $X$, or ones in the column.

(2) The closure of a 1-cell is homeomorphic either to $S^{1}$ or to $D^{1}$. Thus every 1 -index of $n$-dimensional space $X, n \geq 1$, is a two-column matrix, the first column of which consists only of ones and twos; and every $(1,0)$-size of space $X$ is a 3-tuple $\left\langle 0, s_{0}(1), s_{0}(2)\right\rangle_{10}$, where $s_{0}(1)$ and $s_{0}(2)$ are the numbers of 1-cells, whose closures are homeomorphic to $S^{1}$ and $D^{1}$, respectively. 
(3) If space $X$ is cellular equivalent to a two dimensional manifold equipped with a partition, then it has $(2,1)$-size $\left\langle s_{1}(0), s_{1}(1), \ldots, s_{1}\left(p_{21}\right)\right\rangle_{21}$, where $s_{1}(k)$ is the number of 2-cells, which are cellular equivalent to the $k$-gon, $k=0,1,2, \ldots, p_{21}$. In particular, the $(2,1)$-size of the 0 -gon $S^{2}$ is $\langle 1\rangle_{21}$, and if $X$ is different from the 0 -gon, then $s_{1}(0)=0$.

Examples 4.5 (1) If cellular space $X$ is cellular equivalent to the solid torus described in Example 2.4.1, then it has index [1,2,2,1], 0-index [1], 1-index $\left[\begin{array}{ll}1 & 1 \\ 1 & 1\end{array}\right], 2$-index $\left[\begin{array}{lll}1 & 1 & 1 \\ 1 & 2 & 1\end{array}\right]$, and 3-index [ $\left.\begin{array}{llll}1 & 2 & 2 & 1\end{array}\right]$ and has $(k, l)$-sizes $\langle 0,1\rangle_{00},\langle 0,2\rangle_{10},\langle 0,2\rangle_{20},\langle 0,1,1\rangle_{21}$, $\langle 0,1\rangle_{30},\langle 0,0,1\rangle_{31}$, and $\langle 0,0,1\rangle_{32}$.

(2) In Fig. 1, we use a combined approach to order the polyhedra with six faces: first, we order them with respect to cellular code phenomenality, and if the polyhedra have the same cellular code, then we order them with respect to $(2,1)$-size phenomenality. In Fig. 1, the notation $\langle\ldots\rangle_{21}$ is reduced to $\langle\ldots\rangle$. On the other hand the order of the polyhedra only with respect to $(2,1)$-sizes is $1,2,4,6,3,5,7$.

\section{Metrics in the Sets of Permutations}

In this section we define normalized metrics in the sets of permutations, which are used in Sect. 6.2 to estimate deviation of the order of knots in the knot catalogue from their order by $(2,1)$-sizes.

Denote the set of all permutations of numbers $1,2, \ldots, k$ as $\mathcal{S}_{k}$. We reduce notation of permutation $\left(\begin{array}{cccc}1 & 2 & \cdots & k \\ \alpha_{1} & \alpha_{2} & \cdots & \alpha_{k}\end{array}\right)$ to $\left(\alpha_{1}, \ldots, \alpha_{k}\right)$ and denote identity permutation $(1, \ldots, k)$ as $\varepsilon_{k}$. For every two permutations $\alpha=\left(\alpha_{1}, \ldots, \alpha_{k}\right)$ and $\beta=\left(\beta_{1}, \ldots, \beta_{k}\right)$, we define two metrics $d_{1}$ and $d_{2}$ by formulas

$$
d_{1}(\alpha, \beta)=\left|\alpha_{1}-\beta_{1}\right|+\cdots+\left|\alpha_{k}-\beta_{k}\right|
$$

and

$$
d_{2}(\alpha, \beta)=\sqrt{\left(\alpha_{1}-\beta_{1}\right)^{2}+\cdots+\left(\alpha_{k}-\beta_{k}\right)^{2}} .
$$

If $\alpha^{-1}$ is the inverse permutation for $\alpha$, then $d_{i}\left(\varepsilon_{k}, \alpha^{-1}\right)=d_{i}\left(\varepsilon_{k}, \alpha\right)$ for both $i=$ 1,2 .

We define the diameter $D_{i}$ of metric space $\left(\mathcal{S}_{k}, d_{i}\right)$ as $\max _{\alpha, \beta \in P_{k}}\left\{d_{i}(\alpha, \beta)\right\}$. One can check that

$$
D_{1}= \begin{cases}\frac{1}{2}\left(k^{2}-1\right) & \text { if } n=1 \bmod 2, \\ \frac{1}{2} k^{2} & \text { if } n=0 \bmod 2\end{cases}
$$

and

$$
D_{2}=\sqrt{\frac{1}{3}\left(k^{3}-k\right)} .
$$

We define in $\mathcal{S}_{k}$ normalized metrics $\rho_{i}(\alpha, \beta)=\frac{d_{i}(\alpha, \beta)}{D_{i}}$, thus the diameter of each metric space $\left(\mathcal{S}_{k}, \rho_{i}\right)$ is equal to 1 . 
Lemma 5.1 (1) Arithmetic mean $\mu_{k}\left(\rho_{1}\right)$ of distances between permutations in metric space $\left(\mathcal{S}_{k}, \rho_{1}\right)$ is defined by formula

$$
\mu_{k}\left(\rho_{1}\right)= \begin{cases}\frac{2}{3} & \text { if } k=1 \bmod 2, \\ \frac{2}{3}\left(1-\frac{1}{k^{2}}\right) & \text { if } k=0 \bmod 2 .\end{cases}
$$

(2) Mean square $\sigma_{k}\left(\rho_{2}\right)$ of distances between permutations in metric space $\left(\mathcal{S}_{k}, \rho_{2}\right)$ is defined by formula

$$
\sigma_{k}\left(\rho_{2}\right)= \begin{cases}0 & \text { if } k=1 \\ \frac{\sqrt{2}}{2} & \text { if } k \geq 2\end{cases}
$$

Proof (1) The proof comes down to the following direct calculation.

$$
\begin{aligned}
\mu_{k}\left(\rho_{1}\right) & =\frac{1}{(k !)^{2}} \sum_{\alpha \in \mathcal{S}_{k}} \sum_{\beta \in \mathcal{S}_{k}} \rho_{1}(\alpha, \beta)=\frac{1}{k !} \sum_{\alpha \in \mathcal{S}_{k}} \rho_{1}\left(\varepsilon_{k}, \alpha\right)=\frac{1}{k ! D_{1}} \sum_{\alpha \in \mathcal{S}_{k}} d_{1}\left(\varepsilon_{k}, \alpha\right) \\
& =\frac{1}{k ! D_{1}}\left[\sum_{\alpha \in \mathcal{S}_{k}}\left(\left|1-\alpha_{1}\right|+\left|1-\alpha_{2}\right|+\cdots+\left|1-\alpha_{k}\right|\right)\right] \\
& =\frac{1}{k ! D_{1}}\left[(k-1) !\left(\sum_{\alpha_{1}=1}^{k}\left|1-\alpha_{1}\right|+\sum_{\alpha_{2}=1}^{k}\left|1-\alpha_{2}\right|+\cdots+\sum_{\alpha_{k}=1}^{k}\left|1-\alpha_{k}\right|\right)\right] \\
& =\frac{2}{k D_{1}}[(k-1) \cdot 1+(k-2) \cdot 2+\cdots+(k-(k-1)) \cdot(k-1)] \\
& = \begin{cases}\frac{2}{3} & \text { if } k=1 \bmod 2, \\
\frac{2}{3}\left(1-\frac{1}{k^{2}}\right) & \text { if } k=0 \bmod 2 .\end{cases}
\end{aligned}
$$

(2) The proof of the second statement repeats the proof of previous one.

\section{Application to Ordering Curves and Knots}

\subsection{Curves}

Let $L \subset \mathbb{R} P^{2}$ be a line and $A_{n} \subset \mathbb{R} P^{2}$ be a curve diffeomorphic to $S^{1}$ such that $L$ and $A_{n}$ have transversal intersection at $n \geq 1$ points. If $n$ is odd, then $A_{n}$ realizes the nonzero homology class in $H_{1}\left(\mathbb{R} P^{2}\right)$, otherwise $A_{n}$ realizes the zero homology class. For $n=1,2, \ldots, 9$, the topological classification of pairs $\left(\mathbb{R} P^{2}, L \cup A_{n}\right)$ consists of $1,1,1,1,2,3,6,15,26$ classes, respectively. These topology classes are depicted in Figs. 7-12, where we use the Poincaré disk (with identified diametrically opposite points on its boundary) as a model of the projective plane, and the boundary of the disk represents the double of the line $L$.

Each union $L \cup A_{n}$ naturally defines a cellular partition of projective plane $\mathbb{R} P^{2}$. Every partition consists of $n$ 0-cells, which are points of the set $L \cap A_{n}$, of $2 n$ open 1-cells, which are components of the set $\left(L \cup A_{n}\right) \backslash\left(L \cap A_{n}\right)$, and of $n+1$ open 
Fig. 7 Types of pairs

$\left(\mathbb{R} P^{2}, L \cup A_{n}\right)$ for $n=1,2,3,4$

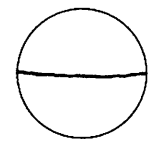

1. $\langle 2\rangle$

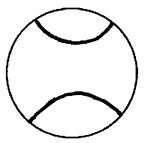

2. $\langle 201\rangle$

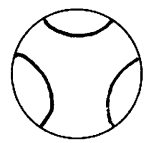

3. $\langle 30001\rangle$

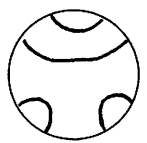

4. $\langle 30101\rangle$

Fig. 8 Types of pairs

$\left(\mathbb{R} P^{2}, L \cup A_{5}\right)$

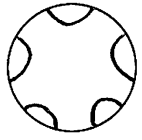

1. $\langle 5(0 \times 7) 1\rangle$

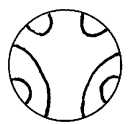

2. $\langle 4020001\rangle$

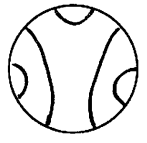

2. $\langle 30201\rangle$

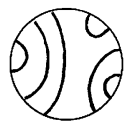

3. $\langle 30301\rangle$

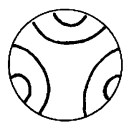

4. $\langle 30301\rangle$

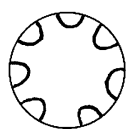

1. $\langle 7(0 \times 11) 1\rangle$

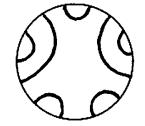

2. $\langle 502(0 \times 5) 1\rangle$

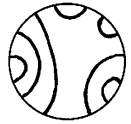

3. $\langle 4030001\rangle$

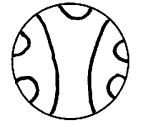

4. $\langle 50003\rangle$

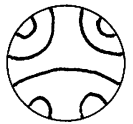

5. $\langle 40202\rangle$

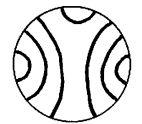

6. $\langle 30401\rangle$

Fig. 10 Types of pairs $\left(\mathbb{R} P^{2}, L \cup A_{7}\right)$

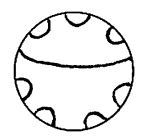

1. $\langle 7(0 \times 5) 101\rangle$

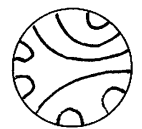

6. $\langle 5020101\rangle$

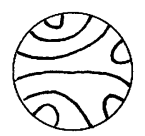

11. $\langle 40302\rangle$

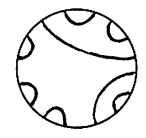

2. $\langle 601010001\rangle$

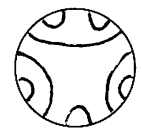

7. $\langle 5020101\rangle$

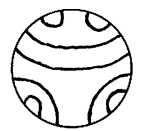

12. $\langle 40302\rangle$

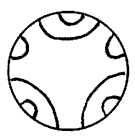

3. $\langle 503(0 \times 5) 1\rangle$

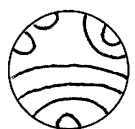

8. $\langle 4040001\rangle$

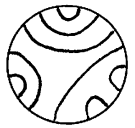

13. $\langle 40302\rangle$

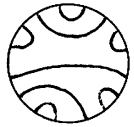

4. $\langle 5020101\rangle$

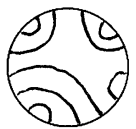

9. $\langle 4040001\rangle$

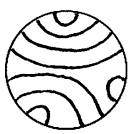

14. $\langle 30501\rangle$
5. $\langle 5020101\rangle$
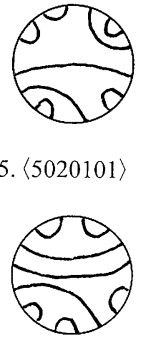

10. $\langle 50103\rangle$

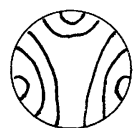

15. $\langle 30501\rangle$

Fig. 11 Types of pairs $\left(\mathbb{R} P^{2}, L \cup A_{8}\right)$

2-cells, which are components of the set $\mathbb{R} P^{2} \backslash\left(L \cup A_{n}\right)$. The cellular space $\mathbb{R} P^{2}$ with such partition has cellular index $[n, 2 n, n+1]$.

If (i) $n \geq 3$, (ii) the curve $A_{n}$ has more than one disjoint connected component diffeomorphic to $S^{1}$, (iii) every component of $A_{n}$ has nonempty transversal intersection with $L$, and (iv) the set $L \cap A_{n}$ consists of $n$ points, then the unions $L \cap A_{n}$ provide other cellular spaces in $\mathbb{R} P^{2}$ of the same index $[n, 2 n, n+1]$. 


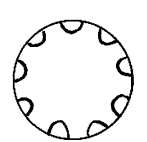

1. $\langle 9(0 \times 15) 1\rangle$

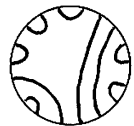

6. $\langle 602010001\rangle$

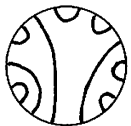

11. $\langle 6010201\rangle$

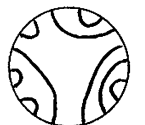

16. $\langle 5030101\rangle$

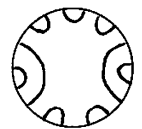

2. $\langle 702(0 \times 9) 1\rangle$

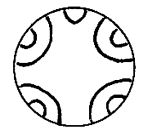

7. $\langle 504(0 \times 5) 1\rangle$

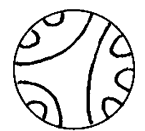

12. $\langle 6010201\rangle$

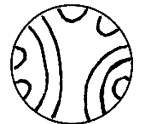

17. $\langle 5030101\rangle$

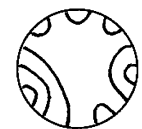

3. $\langle 603(0 \times 7) 1\rangle$

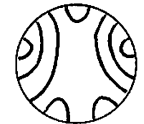

8. $\langle 504(0 \times 5) 1\rangle$

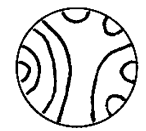

13. $\langle 5030101\rangle$

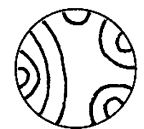

18. $\langle 4050001\rangle$

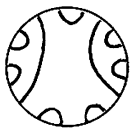

4. $\langle 700020001\rangle$

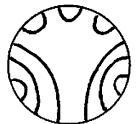

9. $\langle 504(0 \times 5) 1\rangle$

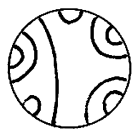

14. $\langle 5030101\rangle$

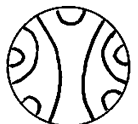

19. $\langle 50203\rangle$

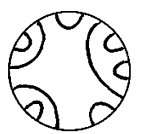

5. $\langle 602010001\rangle$

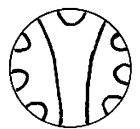

10. $\langle 7000102\rangle$

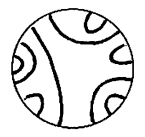

15. $\langle 5030101\rangle$

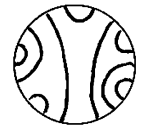

20. $\langle 50203\rangle$

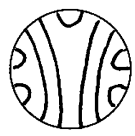

21. $\langle 50203\rangle$

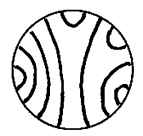

22. $\langle 40402\rangle$

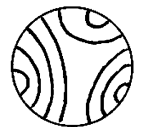

25. $\langle 30601\rangle$

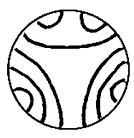

23. $\langle 40402\rangle$

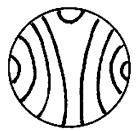

26. $\langle 30601\rangle$

Fig. 12 Types of pairs $\left(\mathbb{R} P^{2}, L \cup A_{9}\right)$

For every cellular space depicted in Figs. 7-12, we calculate its $(2,1)$-size and use them to order these cellular spaces.

Remark 6.1 Since for cellular spaces under consideration $s_{1}(0)=s_{1}(1)=0$, we reduce notations of $(2,1)$-sizes in Figs. $7-18$ from $\left\langle s_{1}(0), s_{1}(1), s_{1}(2), \ldots\right\rangle_{21}$ to $\left\langle s_{1}(2), \ldots\right\rangle$, and since $0 \leq s_{l}(p) \leq 9$, we skip commas between them. And if the zero is repeated $i \geq 4$ times, then we use abbreviation $(0 \times i)$ instead of $0 \cdots 0$.

Let $L_{1}, L_{2} \subset \mathbb{R} P^{2}$ be two lines and $B_{n} \subset \mathbb{R} P^{2}$ be a curve diffeomorphic to $S^{1}$ that satisfies the following properties:

(i) $L_{1}, L_{2}$, and $B_{n}$ have transversal intersection and have no concurrent points,

(ii) each of lines $L_{1}, L_{2}$ intersects curve $B_{n}$ at $n$ points,

(iii) the set $L_{i} \cap B_{n}$, which consists of $n$ points, belongs to one of $n$ connected components of the set $B_{n} \backslash L_{j}$, where $i, j \in\{1,2\}$ and $i \neq j$.

For $n=1,2, \ldots, 6$, the topological classification of pairs $\left(\mathbb{R} P^{2}, L_{1} \cup L_{2} \cup B_{n}\right.$ ) consists of 1, 1, 1, 3, 8, 30 classes, respectively, which are depicted in Figs. 13-17. 
Fig. 13 Types of pairs $\left(\mathbb{R} P^{2}, L_{1} \cup L_{2} \cup B_{n}\right)$, $n=1,2,3$

Fig. 14 Types of pairs $\left(\mathbb{R} P^{2}, L_{1} \cup L_{2} \cup B_{4}\right)$

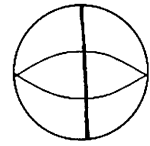

1. $\langle 004\rangle$

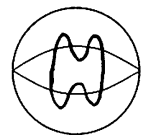

1. $\langle 0620000101\rangle$

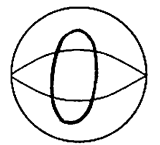

2. $\langle 022006\rangle$

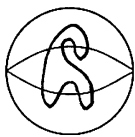

2. $\langle 05111101\rangle$

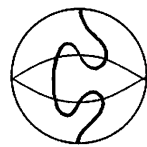

3. $\langle 0410201\rangle$

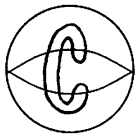

3. $\langle 0413011\rangle$

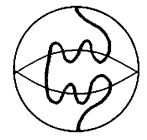

1. $\langle 08100020001\rangle$

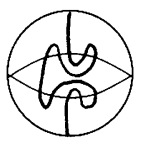

5. $\langle 0612003\rangle$

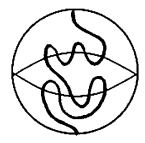

2. $\langle 071110002\rangle$

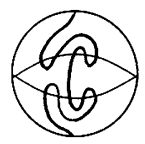

6. $\langle 0602202\rangle$

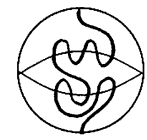

3. $\langle 070120101\rangle$

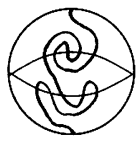

7. $\langle 0513102\rangle$

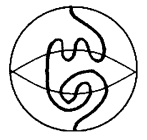

4. $\langle 061210101\rangle$

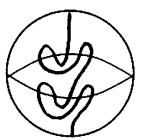

8. $\langle 062202\rangle$

Fig. 15 Types of pairs $\left(\mathbb{R} P^{2}, L_{1} \cup L_{2} \cup B_{5}\right)$

Each union $L_{1} \cup L_{2} \cup B_{n}$ naturally defines a cellular partition of projective plane $\mathbb{R} P^{2}$. Every partition consists of $2 n+10$-cells, which are points of intersections $L_{1} \cap B_{n}, L_{2} \cap B_{n}$, and $L_{1} \cap L_{2}$, of $4 n+2$ open 1-cells, which are components of the set $\left(L_{1} \cup L_{2} \cup B_{n}\right) \backslash\left(L_{1} \cap B_{n}\right) \cup\left(L_{2} \cap B_{n}\right) \cup\left(L_{1} \cap 2_{2}\right)$, and of $2 n+2$ open 2-cells, which are components of the set $\mathbb{R} P^{2} \backslash\left(L_{1} \cup L_{2} \cup B_{n}\right)$. Therefore the cellular space $\mathbb{R} P^{2}$ with such partition has cellular index $[2 n+1,4 n+2,2 n+2]$, and for $n \geq 4$, representatives of topological classes of pairs $\left(\mathbb{R} P^{2}, L_{1} \cup L_{2} \cup B_{n}\right.$ ) realize equiindexial cellular spaces.

If (i) $n \geq 3$, (ii) the curve $B_{n}$ has more than one disjoint connected component diffeomorphic to $S^{1}$, (iii) every component of $B_{n}$ has nonempty transversal intersection either with $L_{1}$ or $L_{2}$, and (iv) each of the sets $L_{1} \cap B_{n}$ and $L_{2} \cap B_{n}$ consists of $n$ points, then the unions $L_{1} \cup L_{2} \cup B_{n}$ provide other cellular spaces in $\mathbb{R} P^{2}$ of the same index $[2 n+1,4 n+2,2 n+2]$.

For every cellular space depicted in Figs. $13-17$, we calculate its $(2,1)$-size and use them to order these cellular spaces. We use the same agreements for notations of $(2,1)$-sizes, which were done in Remark 6.1.

Plane algebraic curves of degree $n$, having connected component of topological types $\left(\mathbb{R} P^{2}, L \cup A_{n-1}\right)$ and $\left(\mathbb{R} P^{2}, L_{1} \cup L_{2} \cup B_{n-2}\right)$ depicted in Figs. $7-17$ often occurred in research on real algebraic geometry (see, for example, [2] and [3, 4] and references in these papers). 

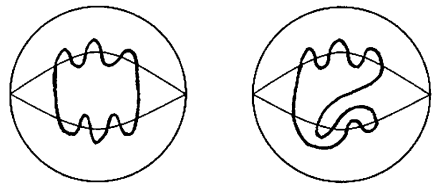

2. $\langle 81210010001\rangle$

1. $\langle 10,2,(0 \times 8), 1,0,1\rangle$

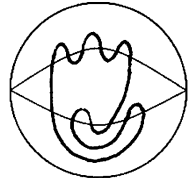

6. $\langle 812100002\rangle$

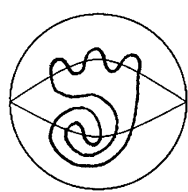

11. $\langle 713100101\rangle$

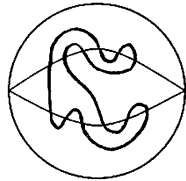

16. $\langle 713011001\rangle$

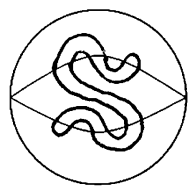

21. $\langle 605200001\rangle$

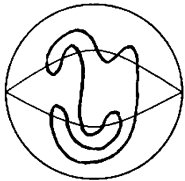

26. $\langle 7130012\rangle$

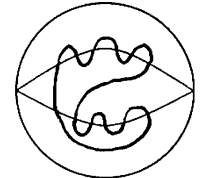

7. $\langle 910011101\rangle$

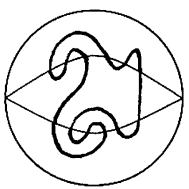

12. $\langle 713100101\rangle$

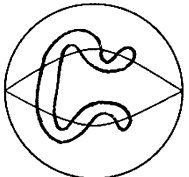

17. $\langle 615001001\rangle$

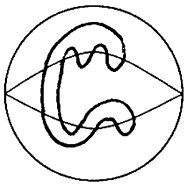

22. $\langle 71301011\rangle$

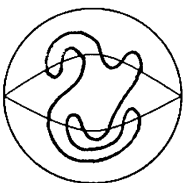

27. $\langle 7032002\rangle$

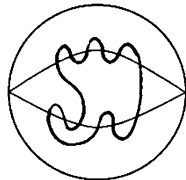

3. $\langle 91110000101\rangle$

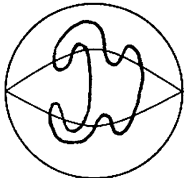

8. $\langle 812001101\rangle$

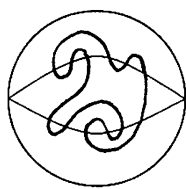

13. $\langle 713100101\rangle$

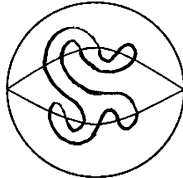

18. $\langle 703210001\rangle$

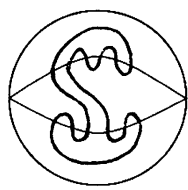

23. $\langle 80113001\rangle$

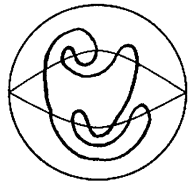

28. $\langle 6141002\rangle$

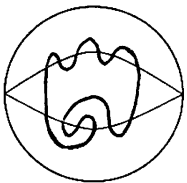

4. $\langle 822(0 \times 5) 101\rangle$

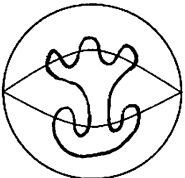

9. $\langle 900210101\rangle$

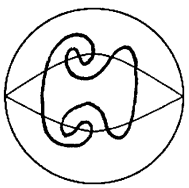

14. $\langle 624000101\rangle$

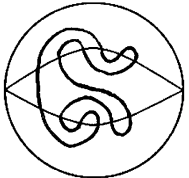

19. $\langle 614110001\rangle$

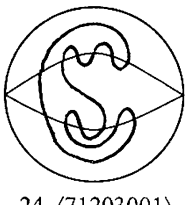

24. $\langle 71203001\rangle$

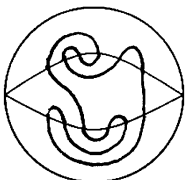

29. $\langle 6141002\rangle$

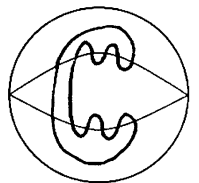

5. $\langle 8110300001\rangle$

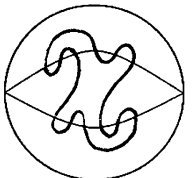

10. $\langle 802200101\rangle$

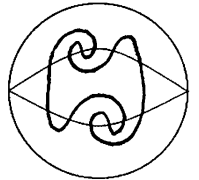

15. $\langle 624000101\rangle$

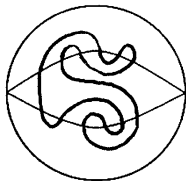

20. $\langle 614110001\rangle$

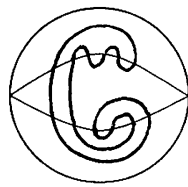

25. $\langle 61402001\rangle$

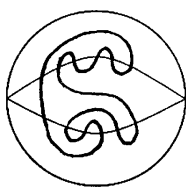

30. $\langle 7120211\rangle$

Fig. 16 Types of pairs ( $\mathbb{R} P^{2}, L_{1} \cup L_{2} \cup B_{6}$ ) (beginning)

\subsection{Ordering Projections of Knots}

Let $K_{n}$ be a knot or link with $n$ crossings, and pr: $K_{n} \rightarrow S^{2}$ be the projection of $K_{n}$ to the standard sphere $S^{2}$ such that image $\operatorname{pr}\left(K_{n}\right)$ has $n$ crossings and has no concurrent points. For $n \geq 3$, image $\operatorname{pr}\left(K_{n}\right)$ defines a cellular partition of the sphere and turns it into cellular space $X\left(K_{n}\right)$. All knots and links with $n$ crossings define equiindexial cellular spaces $X\left(K_{n}\right)$ with cellular index $[n, 2 n, n+2]$. Every such a space $X\left(K_{n}\right)$ has $(0,0)$-size $\langle 0, n\rangle_{00},(1,0)$-size $\langle 0,2 n\rangle_{10}$, and $(1,1)$-size $\langle 0,2 n\rangle_{11}$; but most of spaces $X\left(K_{n}\right)$ have different $(2,1)$-sizes.

We calculate $(2,1)$-sizes of projections of knots with 3 through 9 crossings, and for each number of crossings, we list them in Table 2 regarding to their $(2,1)$-size 


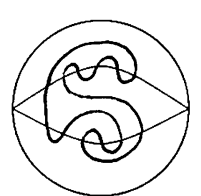

31. $\langle 7120211\rangle$

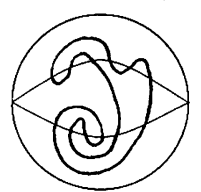

36. $\langle 6140111\rangle$

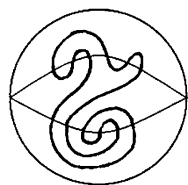

41. $\langle 6042101\rangle$

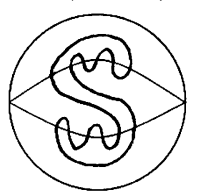

46. $\langle 801032\rangle$

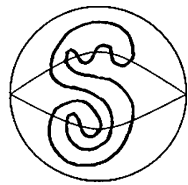

51. $\langle 604121\rangle$

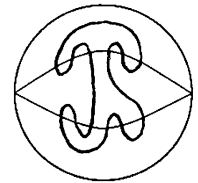

32. $\langle 8011211\rangle$

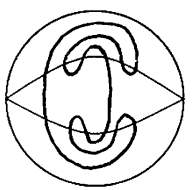

37. $\langle 6140111\rangle$

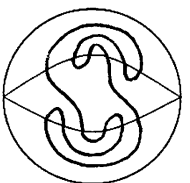

42. $\langle 6042101\rangle$

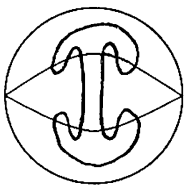

47. $\langle 801032\rangle$

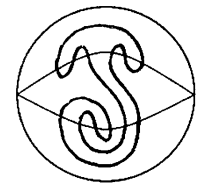

52. $\langle 604121\rangle$

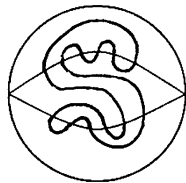

33. $\langle 7031111\rangle$

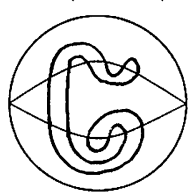

38. $\langle 5160011\rangle$

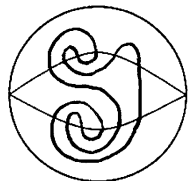

43. $\langle 5151101\rangle$

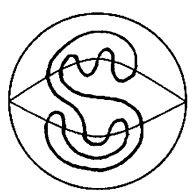

48. $\langle 702131\rangle$

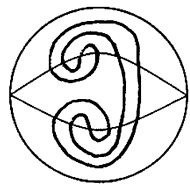

53. $\langle 417011\rangle$

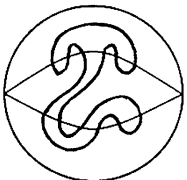

34. $\langle 703111\rangle$

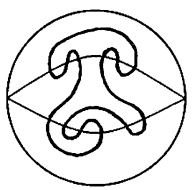

39. $\langle 7022201\rangle$

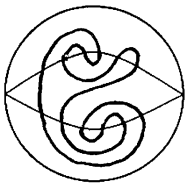

44. 〈5151101〉

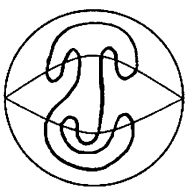

49. $\langle 702131\rangle$

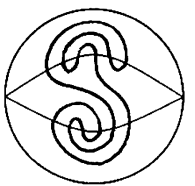

54. $\langle 50522\rangle$

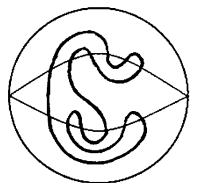

35. $\langle 6140111\rangle$

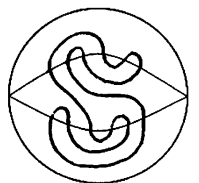

40. $\langle 6042101\rangle$

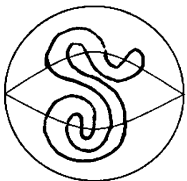

45. $\langle 5062001\rangle$

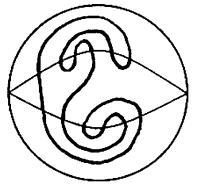

50. $\langle 515021\rangle$

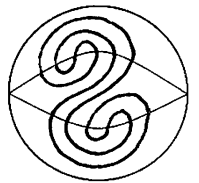

55. $\langle 40721\rangle$

Fig. 17 Types of pairs $\left(\mathbb{R} P^{2}, L_{1} \cup L_{2} \cup B_{6}\right)$ (the end)

phenomenality. We use the same agreements for notations of $(2,1)$-sizes in Table 2 , which we stated in Remark 6.1. If two knots have the same $(2,1)$-size, then we list them by increasing their subscript indices (for example, the $8_{7}$ and $8_{8}$ knots). To complete the exposition, we include the $3_{1}$ and $4_{1}$ knots with their $(2,1)$-sizes in Table 2. Note that the projection of the $0_{1}$ knot does not define cellular partition on sphere $S^{2}$.

It is known that the number $k(n)$ of knots with $n=3,4,5,6,7,8,9$ crossings is equal to $1,1,2,3,7,21,49$, respectively. We assign permutation $\varepsilon_{k(n)}=(1, \ldots, k(n))$ to the order of knots with $n$ crossings provided by their $(2,1)$-sizes. Such order is defined up to the order of knots having the same $(2,1)$-sizes. Every other order of knots with $n$ crossings can be represented by permutation $\gamma^{n}=\left(\gamma_{1}, \ldots, \gamma_{k(n)}\right)$ of the same integers. In particular for knots with 5 through 9 crossings, the knot catalogue provides the following permutations:

$\gamma^{5}=(1,2)$ for knots with 5 crossings,

$\gamma^{6}=(1,2,3)$ for knots with 6 crossings, 
Table 2

\begin{tabular}{llllllllll}
\hline Knot & $(2,1)$-size & Knot & $(2,1)$-size & Knot & $(2,1)$-size & Knot & $(2,1)$-size & Knot & $(2,1)$-size \\
\hline $3_{1}$ & $\langle 32\rangle$ & $8_{5}$ & $\langle 52021\rangle$ & $8_{18}$ & $\langle 082\rangle$ & $9_{12}$ & $\langle 52121\rangle$ & $9_{22}$ & $\langle 4313\rangle$ \\
$4_{1}$ & $\langle 24\rangle$ & $8_{7}$ & $\langle 43111\rangle$ & $9_{1}$ & $\langle 10000009\rangle$ & $9_{36}$ & $\langle 52121\rangle$ & $9_{41}$ & $\langle 3503\rangle$ \\
$5_{1}$ & $\langle 5002\rangle$ & $8_{8}$ & $\langle 4311\rangle$ & $9_{2}$ & $\langle 7200002\rangle$ & $9_{42}$ & $\langle 52121\rangle$ & $9_{16}$ & $\langle 5042\rangle$ \\
$5_{2}$ & $\langle 322\rangle$ & $8_{13}$ & $\langle 34201\rangle$ & $9_{5}$ & $\langle 6210101\rangle$ & $9_{19}$ & $\langle 44021\rangle$ & $9_{29}$ & $\langle 3422\rangle$ \\
$6_{1}$ & $\langle 4202\rangle$ & $8_{3}$ & $\langle 6004\rangle$ & $9_{3}$ & $\langle 702002\rangle$ & $9_{13}$ & $\langle 51311\rangle$ & $9_{30}$ & $\langle 3422\rangle$ \\
$6_{2}$ & $\langle 3311\rangle$ & $8_{6}$ & $\langle 5113\rangle$ & $9_{6}$ & $\langle 603101\rangle$ & $9_{18}$ & $\langle 51311\rangle$ & $9_{43}$ & $\langle 3422\rangle$ \\
$6_{3}$ & $\langle 242\rangle$ & $8_{9}$ & $\langle 422\rangle$ & $9_{8}$ & $\langle 531011\rangle$ & $9_{21}$ & $\langle 43211\rangle$ & $9_{39}$ & $\langle 3341\rangle$ \\
$7_{1}$ & $\langle 700002\rangle$ & $8_{10}$ & $\langle 422\rangle$ & $9_{9}$ & $\langle 603101\rangle$ & $9_{45}$ & $\langle 43211\rangle$ & $9_{48}$ & $\langle 3341\rangle$ \\
$7_{2}$ & $\langle 52002\rangle$ & $8_{11}$ & $\langle 4222\rangle$ & $9_{11}$ & $\langle 522101\rangle$ & $9_{23}$ & $\langle 43211\rangle$ & $9_{49}$ & $\langle 3341\rangle$ \\
$7_{4}$ & $\langle 42201\rangle$ & $8_{12}$ & $\langle 422\rangle$ & $9_{14}$ & $\langle 441101\rangle$ & $9_{26}$ & $\langle 35111\rangle$ & $9_{32}$ & $\langle 2531\rangle$ \\
$7_{3}$ & $\langle 5022\rangle$ & $8_{14}$ & $\langle 3412\rangle$ & $9_{17}$ & $\langle 441101\rangle$ & $9_{27}$ & $\langle 35111\rangle$ & $9_{44}$ & $\langle 2531\rangle$ \\
$7_{5}$ & $\langle 4131\rangle$ & $8_{15}$ & $\langle 3331\rangle$ & $9_{20}$ & $\langle 433001\rangle$ & $9_{31}$ & $\langle 27011\rangle$ & $9_{38}$ & $\langle 326\rangle$ \\
$7_{6}$ & $\langle 3321\rangle$ & $8_{16}$ & $\langle 2521\rangle$ & $9_{35}$ & $\langle 62003\rangle$ & $9_{28}$ & $\langle 34301\rangle$ & $9_{33}$ & $\langle 245\rangle$ \\
$7_{7}$ & $\langle 2511\rangle$ & $8_{17}$ & $\langle 244\rangle$ & $9_{46}$ & $\langle 62003\rangle$ & $9_{37}$ & $\langle 4313\rangle$ & $9_{34}$ & $\langle 164\rangle$ \\
$8_{1}$ & $\langle 620002\rangle$ & $8_{19}$ & $\langle 244\rangle$ & $9_{4}$ & $\langle 70022\rangle$ & $9_{15}$ & $\langle 4313\rangle$ & $9_{47}$ & $\langle 164\rangle$ \\
$8_{2}$ & $\langle 530011\rangle$ & $8_{20}$ & $\langle 244\rangle$ & $9_{7}$ & $\langle 61112\rangle$ & $9_{24}$ & $\langle 4313\rangle$ & $9_{40}$ & $\langle 083\rangle$ \\
$8_{4}$ & $\langle 521101\rangle$ & $8_{21}$ & $\langle 244\rangle$ & $9_{10}$ & $\langle 60221\rangle$ & $9_{25}$ & $\langle 4313\rangle$ & & \\
\hline
\end{tabular}

$\gamma^{7}=(1,2,4,3,5,6,7)$ for 7 knots with crossings,

$$
\begin{aligned}
\gamma^{8} & =(1,2,4,5,7,8,13,3,6,9,10,11,12,14,15,16,17,19,20,21,18)^{-1} \\
& =(1,2,8,3,4,9,5,6,10,11,12,13,7,14,15,16,17,21,18,19,20)
\end{aligned}
$$

for knots with 8 crossings, and $\gamma^{9}=(1,2,5,3,6,8,9,11,14,17,20,35,46,4,7,10,12,36,42,19,13,18,21,45$, $23,26,27,31,28,37,15,24,25,22,41,16,29,30,43,39,48,49,32,44,38$, $33,34,47,40)^{-1}$

$=(1,2,4,14,3,5,15,6,7,16,8,17,21,9,31,36,10,22,20,11,23,34,25,32$, $33,26,27,29,37,38,28,43,46,47,12,18,30,45,40,49,35,19,39,44,24$, 13, 48, 41, 42) for knots with 9 crossings.

One can see that for knots with 5 and 6 crossings, the order of knots in the knot catalogue coincides with the order by $(2,1)$-sizes. In terms of the next paragraph, the deviation for knots with 5 and 6 crossings is zero.

Using normalized metrics $\rho_{i}, i=1,2$, defined in Sect. 5, we calculate the deviations of orders of knots with 7, 8, and 9 crossings in the knot catalogue from their orders by $(2,1)$-sizes and put the results in Table 3 . The range of both metrics $\rho_{i}$ satisfies inequality $0 \leq \rho_{i} \leq 1$. Lemma 5.1 provides that for $n=7,8,9$, the arithmetic mean of deviations in metric space $\left(\mathcal{S}_{k(n)}, \rho_{1}\right)$ is $\mu_{k(n)}\left(\rho_{1}\right)=\frac{2}{3}$, and the mean square of deviations in metric space $\left(\mathcal{S}_{k(n)}, \rho_{2}\right)$ is $\sigma_{k(n)}\left(\rho_{2}\right)=\frac{\sqrt{2}}{2}$. Since inequalities 
Table 3

\begin{tabular}{llll}
\hline$n$ & 7 & 8 & 9 \\
\hline$\rho_{1}\left(\varepsilon_{k(n)}, \gamma^{n}\right)$ & 0.083 & 0.136 & 0.305 \\
$\rho_{2}\left(\varepsilon_{k(n)}, \gamma^{n}\right)$ & 0.134 & 0.177 & 0.366 \\
\hline
\end{tabular}

Fig. 18

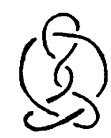

$\langle 4131\rangle$

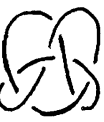

$\langle 2511\rangle$

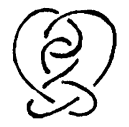

$\langle 324\rangle$

$7 s$

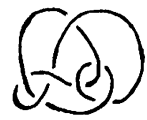

$\langle 243\rangle$

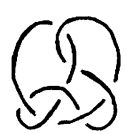

〈3321)

76

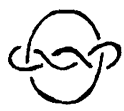

$\langle 323\rangle$
2511〉
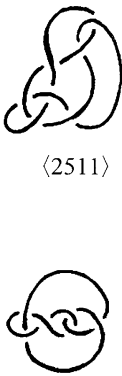

$\langle 242\rangle$

77 $6_{3}{ }^{2}$

$\rho_{1}\left(\varepsilon_{k(n)}, \gamma^{n}\right)<\mu_{k(n)}\left(\rho_{1}\right)$ and $\rho_{2}\left(\varepsilon_{k(n)}, \gamma^{n}\right)<\sigma_{k(n)}\left(\rho_{1}\right)$ hold for $n=5,6,7,8,9$, it makes sense to consider that the order in the knot catalogue is close to the order by (2, 1)-sizes.

The same knot (or link) can have different projections on the standard sphere $S^{2}$ and therefore can define equiindexial cellular spaces, which are not cellular equivalent. In Fig. 18 one can see pairs of projections of the $7_{5}, 7_{6}$, and $7_{7}$ knots and the $6_{3}^{2}$ link with distinct $(2,1)$-sizes. This means that one can define the order relation in the set of all projections of the same knot (or link) by $(2,1)$-sizes and take it into account for ordering knots (or links) with the same number of crossings.

\section{References}

1. Farmer, D.W., Stanford, T.B.: Knots and surfaces: a guide to discovering mathematics. Am. Math. Soc., Ser. Math. World 6, 101 (1996)

2. Korchagin, A.B., Polotovskii, G.M.: On arrangements of a plane real quintic curve with respect to a pair of lines. Commun. Contemp. Math. 5(1), 1-24 (2003)

3. Korchagin, A.B., Smith, D.E.: Patchworking singularities $A_{\mu}$ and $D_{\mu}$ and meanders of their smoothing. In: Zapiski Nauchnykh Seminarov POMI. Notes of Scientific Seminars of Steklov Inst. of Math., St. Petersburg Branch, vol. 299, pp. 193-217 (2003), English

4. Korchagin, A.B., Smith, D.E.: Patchworking singularities $A_{\mu}$ and $D_{\mu}$ and meanders of their smoothing. J. Math. Sci. 131, 5366-5380 (2005)

5. Prokhorov, Y.V. (ed.): Mnogogrannik (Polyhedron), Mathematical Encyclopedic Dictionary, Soviet Encyclopedia (1988), Russian

6. Rolfsen, D.: Knots and Links, p. 439. Publish or Perish Press, Berkeley (1976)

7. Vinogradov, I.M. (ed.): Mathematical Encyclopedia, vol. 5, clm. 484-485, Soviet Encyclopedia (1985), Russian 